\title{
Genetic interactions between the chromosome axis-associated protein Hop1 and homologous recombination determinants in Schizosaccharomyces pombe
}

\author{
Simon David Brown ${ }^{1,2} \cdot$ Olga Dorota Jarosinska ${ }^{1} \cdot$ Alexander Lorenz $^{1}$ (D)
}

Received: 8 March 2018 / Accepted: 14 March 2018 / Published online: 17 March 2018

(c) The Author(s) 2018

\begin{abstract}
Hop1 is a component of the meiosis-specific chromosome axis and belongs to the evolutionarily conserved family of HORMA domain proteins. Hop1 and its orthologs in higher eukaryotes are a major factor in promoting double-strand DNA break formation and inter-homolog recombination. In budding yeast and mammals, they are also involved in a meiotic checkpoint kinase cascade monitoring the completion of double-strand DNA break repair. We used the fission yeast, Schizosaccharomyces pombe, which lacks a canonical synaptonemal complex to test whether Hop1 has a role beyond supporting the generation of double-strand DNA breaks and facilitating inter-homolog recombination events. We determined how mutants of homologous recombination factors genetically interact with hopl, studied the role(s) of the HORMA domain of Hop1, and characterized a bio-informatically predicted interactor of Hop1, Aho1 (SPAC688.03c). Our observations indicate that in fission yeast, Hop1 does require its HORMA domain to support wild-type levels of meiotic recombination and localization to meiotic chromatin. Furthermore, we show that hop $1 \Delta$ only weakly interacts genetically with mutants of homologous recombination factors, and in fission yeast likely has no major role beyond break formation and promoting inter-homolog events. We speculate that after the evolutionary loss of the synaptonemal complex, Hop1 likely has become less important for modulating recombination outcome during meiosis in fission yeast, and that this led to a concurrent rewiring of genetic pathways controlling meiotic recombination.
\end{abstract}

Keywords Schizosaccharomyces pombe $\cdot$ Meiosis $\cdot$ Homologous recombination $\cdot$ Chromosome axis $\cdot$ Hop1

\section{Introduction}

Chromosomes of diploid organisms are present in two sets, each derived from one of the parents. To reproduce sexually, this genetic material is halved exactly to produce haploid

Communicated by M. Kupiec.

Electronic supplementary material The online version of this article (https://doi.org/10.1007/s00294-018-0827-7) contains supplementary material, which is available to authorized users.

Alexander Lorenz

a.lorenz@abdn.ac.uk

1 Institute of Medical Sciences (IMS), University of Aberdeen, Foresterhill, Aberdeen AB25 2ZD, UK

2 Present Address: MRC Institute of Genetics and Molecular Medicine, University of Edinburgh, Edinburgh EH4 2XU, UK cells that contain only one chromosome set, the gametes. During fertilization, two gametes from different sexes fuse to form a zygote, re-establishing a double chromosome set. A specialized kind of cell division, called meiosis, is employed to halve the genetic material. In meiosis, one round of chromosome duplication is followed by two rounds of chromosome segregation. Remarkably, correct segregation of chromosomes in meiosis requires formation of doublestrand DNA breaks (DSBs) by the conserved transesterase Spo11 (Lam and Keeney 2015), followed by repair through homologous recombination. Homologous recombination, in combination with sister chromatid cohesion, establishes physical connections (chiasmata) between maternal and paternal chromosomes that guide their proper segregation, and promotes reciprocal exchange (crossovers) of maternal and paternal genetic information (Hunter 2015). Careful regulation of this complex process is imperative to ensure the required number of physical links between the correct 
partner chromosomes, and that unrepaired DNA breaks are mended (Lam and Keeney 2015; Hunter 2015).

The repair of the Spo11-induced DSBs is started by DNA resection. During the initial step of DNA resection Spo11, which is covalently linked to the DNA ends, is endonucleolytically removed by the Mre11-Rad50-Nbs1 (Mre11-Rad50-Xrs2 in Saccharomyces cerevisiae) complex in cooperation with Ctp1 (Sae2 in S. cerevisiae) (Neale et al. 2005; Hartsuiker et al. 2009). Long-range resection then exposes substantial $3^{\prime}$ tails which are used to invade homologous template DNA on the homologous chromosome or the sister chromatid (strand exchange) to mend the DSB by recombination (reviewed in Mimitou and Symington 2011; Hunter 2015; Lorenz 2017). Key factors for the strand exchange process in meiosis are the RecA-type recombinases Rad51 and Dmc1 (Shinohara et al. 1997; Grishchuk and Kohli 2003) supported by their mediators Rad55-Rad57, Swi5-Sfr1, and Rlp1-Rdl1-Sws1 (SHU complex in S. cerevisiae) (Tsutsui et al. 2001; Grishchuk and Kohli 2003; Akamatsu et al. 2003; Martín et al. 2006; Sasanuma et al. 2013; Hong et al. 2013; Lorenz et al. 2014). These Rad51/Dmc1-mediators are important for generating and stabilizing Rad51/Dmc1-coated nucleoprotein filaments which then invade homologous template DNA (Sung 1997; Haruta et al. 2006). The DNA on the homologous chromosome (or sister chromatid) being invaded is opened as a displacement loop (D-loop), which can then be extended by DNA synthesis using the invading sequence as primer (reviewed in Brown and Bishop 2014). The way in which D-loops or recombination intermediates derived from it are processed determines whether repair will occur as a crossover (CO) or a non-CO, and Rad51/Dmc1-mediators play an important role in this (reviewed in Brown and Bishop 2014; Hunter 2015; Lorenz 2017).

In fission yeast, processing of most meiotic recombination intermediates into $\mathrm{CO}$ and some non-COs depends on the structure-selective endonuclease Mus81-Eme1, the lack of which results in meiotic catastrophe and strongly reduced spore viability (Osman et al. 2003; Smith et al. 2003). Additionally, a sizeable fraction of non-COs is produced by the action of the FANCM-ortholog Fml1, a DEAD/DEAH-box DNA helicase, and it has been shown that Mus81-Eme1 and Fml1 work in parallel to process meiotic recombination intermediates (Lorenz et al. 2012). In a wild-type situation Fml1 action seems to be curtailed by Rad51/Dmc1mediators, because weakening the strand exchange reaction by removing any Rad51/Dmc1-mediator in a mus $81 \Delta$ background ameliorates the strong spore viability defect of mus81 $\Delta$ without restoring CO formation; this rescue depends on the presence of Fml1 (Lorenz et al. 2012, 2014).

To facilitate the generation of DSBs and their correct repair during meiosis, chromosomes undergo a progression of changes starting with the establishment of meiosis-specific chromosome axes and, in most organisms, culminating in the formation of the synaptonemal complex - an elaborate proteinaceous structure (reviewed in Cahoon and Hawley 2016). Components of the synaptonemal complex seem to be critical for controlling several aspects of meiotic chromosome metabolism, from pairing of homologous chromosomes, to initiation of recombination and maturation of the physical interactions between the homologous chromosomes (reviewed in Cahoon and Hawley 2016; Gray and Cohen 2016). However, some organisms have overcome the necessity for a fully fledged synaptonemal complex and perform meiosis with a simplified version of it (reviewed in Loidl 2016). The fission yeast Schizosaccharomyces pombe is the best-studied organism executing meiosis without a canonical synaptonemal complex (reviewed in Loidl 2006).

Despite lacking a synaptonemal complex, fission yeast meiotic chromosomes assemble axes, called linear elements (Olson et al. 1978; Bähler et al. 1993; Molnar 2003), consisting of proteins which are evolutionarily related to axial/lateral elements of the synaptonemal complex (Lorenz et al. 2004), and building upon the existing mitotic chromosome axis organization (reviewed in Ding et al. 2016; Iwasaki and Noma 2016). One of these components is Hop 1 which, in contrast to other meiotic chromosome axis factors, is conserved on the protein sequence level with homologs from yeast to man (Lorenz et al. 2004; Rosenberg and Corbett 2015). In both S. cerevisiae and Sz. pombe, Hop1 localizes to the meiosis-specific chromosome axis (Hollingsworth et al. 1990; Smith and Roeder 1997; Lorenz et al. 2004), and deletion of HOPl/hopl ${ }^{+}$causes a decrease in meiotic recombination and homologous chromosome pairing (Hollingsworth and Byers 1989; Loidl et al. 1994; Latypov et al. 2010). Hop1 is characterized by a conserved N-terminal HORMA (op 1-ㄹev7-Mad2) domain (Aravind and Koonin 1998) (Fig. 1a) and is the founding member of the meiotic HORMA domain (HORMAD) family proteins. In contrast to yeasts which contain only one meiotic HORMAD (Hop1), higher eukaryotes contain multiple paralogs of meiotic HORMADs: these include HIM-3, HTP-1, HTP-2, and HTP-3 in C. elegans; ASY1 and ASY2 in Arabidopsis; and HORMAD1 and HORMAD2 in mammals (Zetka et al. 1999; Caryl et al. 2000; Couteau and Zetka 2005; Martinez-Perez and Villeneuve 2005; Goodyer et al. 2008; Fukuda et al. 2010). In fungi, plants, and mammals, the HORMA domain is followed by several [S/T]Q residues representing potential Tel1/ATM-Rad3/Mec1/ATR phosphorylation sites, which enable meiotic HORMADs to act as structural adaptors for a meiotic checkpoint kinase cascade (Sanchez-Moran et al. 2007; Carballo et al. 2008; Daniel et al. 2011; Kogo et al. 2012; Osman et al. 2018) (Fig. 1a). Fungal Hop1 proteins also contain a centrally located $\mathrm{CxxC} \mathrm{Zn}$-finger motif 


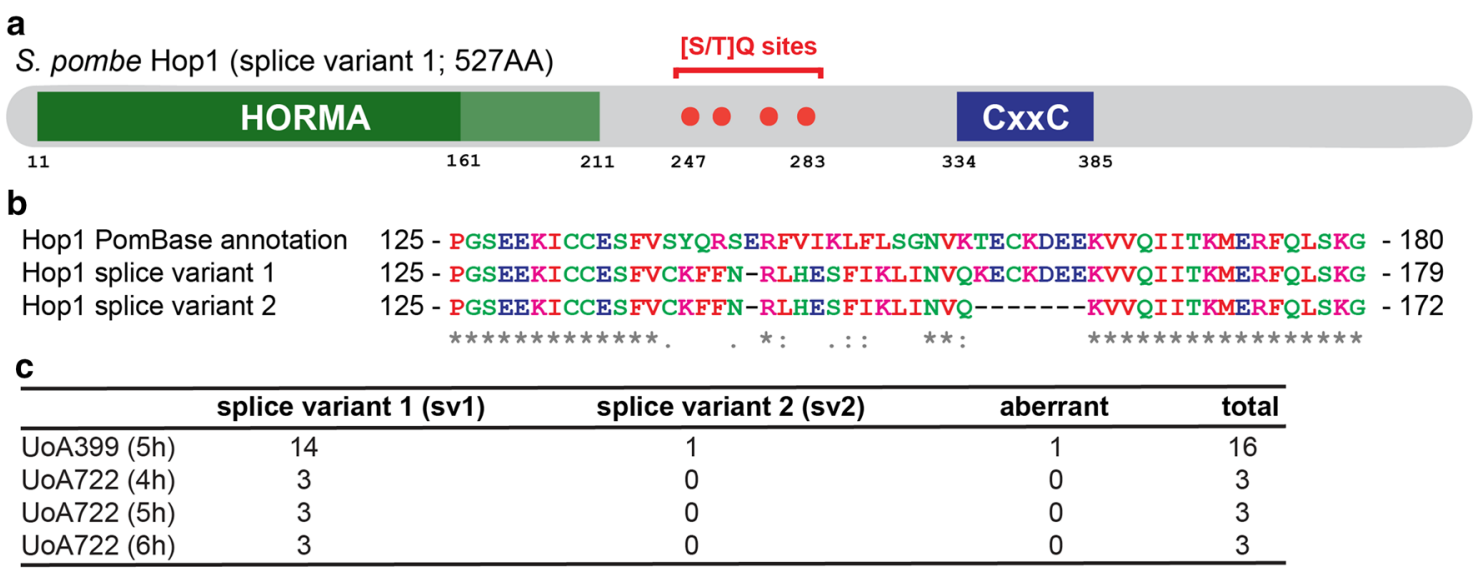

Fig. 1 Schizosaccharomyces pombe Hop1 domain structure and alignment between annotated and experimentally determined versions. a Schematic of the domain structure of the experimentally derived sv1 (splice variant 1) version of Hop1 with the HORMA domain at the $\mathrm{N}$ terminus (AAs 11-211) and four potential [S/T]Q phosphorylation sites (red dots) distributed between AAs 247-283 and residues 334-385 comprising the CxxC Zn-finger domain (dark blue). The HORMA domain can be subdivided into a core domain (dark green) represented by the most C-terminal 150 AAs and a "safety-belt" region (light green; residues 161-211) (Rosenberg and Corbett 2015; West et al. 2017). Positions of key AA residues are indicated below the schematic. b Clustal $\Omega$ alignment (Sievers et al. 2014) of part of the HORMA domain (starting at residue 125) of the

(Hollingsworth et al. 1990) (Fig. 1a) which has been demonstrated to be important for in vitro DNA-binding of $S$. cerevisiae Hop1 (Tripathi et al. 2007). Despite its in vitro DNA-binding capabilities, Hop1 requires the presence of Rec10 (Red1 in S. cerevisiae) to localize to chromosome axes in vivo (Smith and Roeder 1997; Lorenz et al. 2004); indeed, Hop1 and Rec10/Red1 have been shown to physically interact with each other (de los Santos and Hollingsworth 1999; Spirek et al. 2010). Rec10 in turn is recruited to chromosome axes by the meiosis-specific cohesin subunit Rec11 upon phosphorylation by casein kinase 1 (Phadnis et al. 2015; Sakuno and Watanabe 2015).

Here, we investigate the genetic interactions of hopl with meiotic recombination determinants in fission yeast to test whether hopl has (a) role(s) beyond its established function in promoting DSB formation (Latypov et al. 2010). Genetic interactions with factors important for recombination intermediate processing ( $m u s 81$ and $f m l l$ ) suggested a potential, likely indirect, role for Hop 1 in the strand exchange process. However, subsequent epistasis analysis with the strand exchange factor $d m c 1$ and Rad51/Dmc1-mediators (rad55, $s f r l$, and $r l p l$ ) did not support the hypothesized separate function of Hop1 in strand exchange. Furthermore, we also show that the HORMA domain is required for wild-type levels of meiotic recombination, and for localization to meiotic chromatin in $S z$. pombe. Implications of the evolutionary current PomBase-annotation of Hop1, and the two observed splice variants Hop1-sv1 and Hop1-sv2. Coloring of AAs follows the standard Clustal $\Omega$ scheme according to physicochemical properties: red for small and hydrophobic AAs (AVFPMILW); blue for acidic AAs (DE); magenta for basic AAs (RK); and green for AAs containing hydroxyl-, sulfhydryl-, or amine-groups. Consensus symbols below the alignment indicate full conservation $(*)$ and conservation of groups with strongly similar (:) or weakly similar (.) properties; for details see http://www.ebi.ac.uk/Tools/msa/clustalo/help/faq.html. c Relative abundance of different hopl splice variants found in cDNA of the indicated diploid strains at the given time points of a meiotic time course

differences regarding meiotic HORMAD proteins and their interactions are discussed.

\section{Materials and methods}

\section{Schizosaccharomyces and E. coli culture conditions}

E. coli strains were grown on LB and SOC media-where appropriate containing $100 \mu \mathrm{g} / \mathrm{ml}$ ampicillin (Sambrook and Russell 2000). Competent cells of $E$. coli strains NEB $10^{\circledR}$-beta (New England BioLabs Inc., Ipswich, MA, USA) and XL1-blue (Agilent Technologies, Santa Clara, CA, USA) were transformed following the protocols provided by the manufacturers.

Schizosaccharomyces strains used for this study are listed in Supplementary Table S1. Yeast cells were cultured on yeast extract (YE), yeast nitrogen base glutamate (YNG) and Edinburgh minimal media with glutamate as nitrogen source (EMMG) agar plates containing the required supplements (concentration $250 \mathrm{mg} / \mathrm{l}$ on YE, $75 \mathrm{mg} / \mathrm{l}$ on YNG and EMMG). Crosses were performed on malt extract (ME) agar containing the required supplements at a final concentration of $50 \mathrm{mg} / \mathrm{l}$, unless strains to be crossed contained plasmids, in which case they were sporulated on sporulation agar with supplement (SPAS) plates with the necessary supplements at 
$50 \mathrm{mg} / \mathrm{l}$ to maintain selection for the plasmids (Smith 2009; Sabatinos and Forsburg 2010). Transformation of yeast strains was done using a standard lithium acetate procedure (Keeney and Boeke 1994) with the modifications described in Brown and Lorenz (2016).

To obtain meiotic $S z$. pombe cells for chromatin spreading and cDNA preparation, meiotic time courses were executed according to a published protocol (Loidl and Lorenz 2009).

\section{Yeast strain and plasmid construction}

For specifics of strain and plasmid construction, please refer to Supplementary Materials.

Construction of the kanMX6-marked hop $1 \Delta-1$ allele (Latypov et al. 2010) and the kanMX6-marked mug20 ${ }^{+}-$ GFP (Estreicher et al. 2012) have been described previously. The natMX6-marked hop $1 \Delta-25$ was derived from hop $1 \Delta-1$ using an established marker swap protocol (Sato et al. 2005; Lorenz 2015).

To produce hopl-alleles expressing a particular splice variant or the hop 1- $\triangle H O R M A D$ mutant, we first partially deleted hopl retaining the coding sequence directly upstream of the first and directly downstream of the last intron using a ura4 marker (Grimm et al. 1988). The resulting strain carrying the hop 1-3 allele (UoA725) was subsequently transformed with the cDNA of one of the two splice variants or a hopl- $\triangle H O R M A D$ construct; candidate strains were selected by resistance to FOA (5-fluoroorotic acid) and then subjected to further testing.

Epitope tagging of Hop1 with 13 myc was achieved by cloning a transformation cassette into pFA6a-13myckanMX6 (Bähler et al. 1998) with sequences from the 3' end and downstream flanking region of the hopl coding sequence. This transformation cassette was amplified by PCR and transformed into strains UoA746, UoA868, and UoA938.

A deletion cassette for ahol was constructed by cloning flanking sequences of SPAC688.03c into pAG25 upand downstream of the natMX4 marker (Goldstein and McCusker 1999). The resulting plasmid (pALo116) was linearized by a restriction digest and transformed into the standard laboratory strain FO652.

To overexpress $a h o 1^{+}$, the coding sequence of SPAC688.03c was amplified by PCR and cloned into pREP41-eGFPC (Craven et al. 1998).

To construct plasmids for overexpression of $h_{o p I^{+}}$in $S z$. pombe, we cloned PCR products representing genomic or cDNA versions of the hopl coding sequence into pJR41XL (Moreno et al. 2000). During DNA sequencing, we found discrepancies with the hopl coding sequence prediction on PomBase (https://www.pombase.org/, last accessed 03/06/2017) (see "Results" section for details). To corroborate this initial finding, we also cloned meiotic cDNA clones from two independently constructed strains (UoA399 and UoA722) into pUC8.

All plasmid constructs (for sequences see supporting online material, Lorenz 2018), as well as epitope-tagged, splice variant, and hopl- $\triangle H O R M A D$ strains were verified by DNA sequencing (Source BioScience plc, Nottingham, UK).

All DNA modifying enzymes (high-fidelity DNA polymerase Q5, restriction endonucleases, T4 DNA ligase) and NEBuilder HiFi DNA Assembly Master Mix were obtained from New England BioLabs. Oligonucleotides were supplied by Sigma-Aldrich Co. (St. Louis, MO, USA).

\section{Genetic and molecular biology techniques}

Spot assays to monitor growth and genotoxin sensitivities were essentially performed as described previously (Doe et al. 2000; Lorenz et al. 2009). Spot assays of deletion strains were done on YE plates, and of strains harboring plasmids on EMMG plates containing the required supplements. To repress gene expression from pREP41-type plasmids in strains grown on EMMG, thiamine was added to a final concentration of $4 \mu \mathrm{M}$ (Sabatinos and Forsburg 2010).

Genetic recombination assays and spore viability testing by random spore analysis have been described in detail (Osman et al. 2003; Lorenz et al. 2010, 2012, 2014; Sabatinos and Forsburg 2010).

Meiotic cDNA was obtained from cells undergoing synchronized azygotic wild-type meiosis. Aliquots of cell culture were drawn at several time points during a meiotic time course by first extracting RNA using the NucleoSpin TriPrep kit (Macherey-Nagel GmbH \& Co. KG, Düren, Germany) and then reverse-transcribing the RNA into cDNA using the ProtoScript II First Strand cDNA Synthesis kit (New England BioLabs) following the instructions of the respective manufacturer.

\section{Chromatin spreads, immunocytochemistry, and microscopy}

Nuclear spreads of meiotic $S z$. pombe cells were prepared as described previously (Loidl and Lorenz 2009), with the only modification that Lallzyme MMX (Lallemand Inc., Montréal, Canada) at a final concentration of $100 \mathrm{mg} / \mathrm{ml}$ was used as the sole enzyme in the spheroplasting solution (Flor-Parra et al. 2014).

Immunostaining was performed as previously described (Loidl and Lorenz 2009) using polyclonal rabbit $\alpha$-myc (ab9106; Abcam PLC, Cambridge, UK) at a 1:500 dilution and monoclonal rat $\alpha$-GFP [3H9] (ChromoTek GmbH, Planegg-Martinsried, Germany) at a 1:100 dilution as primary antibodies. Antibody-bound protein was visualized using donkey $\alpha$-rabbit IgG AlexaFluor-555 (ab150062; Abcam) and donkey $\alpha$-rat IgG AlexaFluor-488 (ab150153; 
Abcam), both at a 1:500 dilution, as secondary antibodies conjugated to fluorophores. DNA was stained by Hoechst 33342 (Molecular Probes, Eugene, OR, USA) at a final concentration of $1 \mu \mathrm{g} / \mathrm{ml}$.

Analysis was done using a Zeiss Axio Imager.M2 (Carl Zeiss AG, Oberkochen, Germany) epifluorescence microscope equipped with the appropriate filter sets to detect red, green, and blue fluorescence. Black-and-white images were taken with a Zeiss AxioCam MRm CCD camera controlled by AxioVision 40 software v4.8.2.0. Images were pseudocolored and overlaid using Adobe Photoshop CC (Adobe Systems Inc., San José, CA, USA).

\section{Data presentation and statistics}

Data presented as box-and-whisker plots were created in RStudio 1.0.136 (R version 3.3.1) (http://www.r-proje ct.org/) using the boxplot() function with its standard settings. The lower and upper 'hinges' of the box represent the first and third quartile, respectively, and the black bar within the box indicates the median (= second quartile). The 'whiskers' represent the minimum and maximum of the range, unless they differ by more than 1.5 times the interquartile distance from the median. In the latter case, the borders of the 1.5 times interquartile distance around the median are indicated by the 'whiskers' and values outside this range ('outliers') are shown as open circles. Raw data and $\mathrm{R}$ scripts used for the box plot() function are available online as supporting material (Lorenz 2018).

Initially, all data involving multiple comparisons underwent an overall statistical assessment using a Kruskal-Wallis one-way analysis of variance to verify that there indeed are statistically significant differences $(\alpha<0.05)$ between data sets within one experiment. All direct one-on-one statistical comparisons between control and experimental data were done using a two-tailed Mann-Whitney $U$ test. Both Kruskal-Wallis and Mann-Whitney $U$ tests are non-parametric and therefore do not assume normal distribution of data points within a data set. Kruskal-Wallis analyses were performed in IBM SPSS Statistics 24.0.0.0 (International Business Machines Corporation, Armonk, NY, USA), and Mann-Whitney $U$ tests in G*Power 3.1.9.2 (Faul et al. 2007, 2009). $P$ values listed in Supplementary Tables S2 and S3 were determined with a statistical power $(1-\beta)$ set to 0.8 .

\section{Results}

\section{Correction of the Sz. pombe hop1 coding sequence}

When constructing plasmids to overexpress hopl $^{+}$in fission yeast cells, sequencing of our cDNA clones revealed major discrepancies compared to the annotated sequence on PomBase (https://www.pombase.org/, last accessed 03/06/2017). These differences resulted from the incorrect annotation of the position of the third intron (Figs. 1b, $\mathrm{S} 1, \mathrm{~S} 2$ ). Additionally, we detected two splice variants (sv) of hopl (https://www.ebi.ac.uk/ena/data/view/LT963776; https://www.ebi.ac.uk/ena/data/view/LT907816). The main cDNA version (hopl-svl) translated into a protein with 527 amino acids (AAs) in length which is one AA shorter than the currently annotated one (528 AAs). The discrepancy affects AAs 138-156/157 in the core region of the HORMA domain (Fig. 1). A second, shorter splice variant (hop 1-sv2) seems to use a downstream AG as an alternative splice acceptor site at the third intron, thereby expanding it by another 21 bps (Figs. 1b, S1, S2). In a total of $25 \mathrm{cDNA}$ clones from meiotic cDNA preparations of two independently constructed strains drawn from different time points of a meiotic time course, 23 were of the hopl-svl type, 1 conformed to the hopl-sv2 type, and 1 was aberrant representing a rather short form of hopl with a frameshift (Fig. 1c). Considering the scarcity of hop $1-s v 2$ in our samples, it could well represent a splicing accident or even a PCR artefact.

Using Clustal $\Omega$ (Sievers et al. 2014) the translation of the experimentally determined Hop1-sv1 HORMA domain sequence also produces a slightly better alignment with Hop1 HORMA domains of other Schizosaccharomyces (Fig. S3) species than the predicted one. The region of discrepancy in the experimentally derived $S z$. pombe Hop1 HORMA domain (VCKFFNRLHESFIKLINVQKE) aligns without much gapping (only one AA position in Sz. octosporus and Sz. cryophilus shows a gap) and 7 fully conserved or strongly similar positions to their counterparts in the other Schizosaccharomyces species. The same region from the predicted $S z$. pombe Hop1 (VSYQRSERFVIKLFLSGNVKTE) produces a large gap in the alignment with the $S z$. japonicus sequence, receives a three AAs gap compared to Sz. octosporus and Sz. cryophilus, and aligns with only four fully conserved or strongly similar positions. Over the whole length of the HORMA domain in this multi-species comparison, this results in 10 fully conserved and 34 strongly similar AA residues in the alignment for the predicted $S z$. pombe Hop1 sequence, and in 11 fully conserved and 35 strongly similar AA residues with the experimentally derived one (Fig. S3). This demonstrates that the current annotation of the hopl open 
reading frame on PomBase (https://www.pombase.org/) is erroneous, and that the long, predominant splice variant (hopl-sv1; https://www.ebi.ac.uk/ena/data/view/LT963 776) we identified in our cDNA clones is the correct transcript of hopl.

\section{The long hop1 splice variant hop1-sv1 is sufficient for meiotic recombination}

We were interested in establishing whether the long, predominant version of Hop1 (Hop1-sv1) is fully functional, and whether solely expressing the rare short version Hop1sv2 (lacking 7 AAs in the HORMA domain; see Fig. 1) causes a phenotype. Additionally, we constructed a Hop1 version which lacks the HORMA domain altogether, Hop1$\triangle$ HORMAD. In S. cerevisiae, removing the N-terminal 268 AAs of Hop 1-which includes the HORMA domain-has previously been shown to abolish spore viability completely; however the C-terminal fragment remaining is still capable of binding DNA in vitro (Khan et al. 2013). In contrast, the $M 127 \mathrm{~K}$ point mutation in the centre of the HORMA domain of C. elegans HTP-1, one of four meiotic HORMAparalogs in the nematode, results in only a minor meiotic phenotype despite HTP- $1^{\mathrm{M} 127 \mathrm{~K}}$ not localizing to meiotic chromosome axes (Silva et al. 2014). The Sz. pombe short form hop 1-sv2 is apparently not strongly expressed (if not a splicing accident or PCR artefact) in a wild-type background and thus unlikely to play a (major) role. However, in light of the distinct phenotypes of HORMA-domain mutants in budding yeast and $C$. elegans, we wanted to use the hop 1$s v 2$ variant and the hopl- $\triangle H O R M A D$ mutant to probe the role of the HORMA domain for meiotic recombination and Hop1 localization to meiotic chromatin in fission yeast. The 7 AAs (positions 157-163) missing in Hop1-sv2 are close to the boundary between the HORMA core domain and the HORMA "safety-belt" region (Figs. 1a, b, S2) (Rosenberg and Corbett 2015; West et al. 2017), and their loss might affect interaction of Hop1 with the meiotic chromosome axis similar to nematode HTP- $1^{\mathrm{M} 127 \mathrm{~K}}$ and also interfere with Hop1's self-oligomerization. The hopl- $\triangle H O R M A D$ strains serve as controls, to monitor the effect removal of the complete HORMA domain has on meiotic recombination and Hop1 localization.

Previously, it has been shown that the full deletion of hopl causes a reduction in both intragenic (gene conversion) and intergenic (crossover) recombination at several genetic intervals in fission yeast with a concomitant increase in intrachromosomal recombination (Latypov et al. 2010). The hopl deletion also leads to defects in homologous pairing along chromosome I during meiosis (Latypov et al. 2010). These phenotypes can at least be partially explained by a reduction in DSB formation and a preponderance of DSBs being repaired from the sister chromatid rather than the homolog (Rothenberg et al. 2009; Latypov et al. 2010). Using our meiotic recombination assay system (Lorenz et al. 2010), which allows us to concomitantly measure spore viability, and the frequency of gene conversion (GC), crossovers (COs) and COs with GC events, we found a statistically significant decrease in all recombination measures in a hop $1 \Delta$ compared to a wild-type cross (Fig. 2). Indeed, GC decreased 4.7-fold $\left(p=4.53 \times 10^{-13}\right)$ (Fig. 2b), COs 2.8fold $\left(p=3.61 \times 10^{-6}\right)$ (Fig. $\left.2 \mathrm{c}\right)$, and COs associated with GC events were reduced by 8.7 percentage points $(p=0.01)$ (Fig. 2d) (see also Table S2). Overall, this reduction in recombination outcome did not negatively affect spore viability (Table S2). Recombination of a strain expressing only the long, predominant version hopl-svl (from the hoplpromoter at its original locus without any marker genes inserted) was indistinguishable from wild type, whereas replacing the wild-type copy of hopl with hopl-sv2 led to a moderate reduction in GC (1.4-fold, $\left.p=1.51 \times 10^{-3}\right)$ and COs (1.7-fold, $\left.p=2.27 \times 10^{-3}\right)$, but not in COs associated with GC events $(p=0.76)$ compared to wild type (Fig. 2, Table S2). The hop 1- $\triangle H O R M A D$ in turn was indistinguishable from a full deletion (Fig. 2, Table S2). These results indicate that in line with hopl-svl being the predominant, if not the exclusive, splice variant behaves like wild-type hopl containing all its introns, that hopl-sv2 lacking 21 nucleotides in the HORMA domain is not fully functional producing a hypomorphic phenotype, and that removal of the complete HORMA domain (hopl- $\triangle H O R M A D$ ) produces a null phenotype.

\section{Hop 1 localizes to meiotic chromatin and forms linear elements independent of a full-length HORMA domain}

As with organisms possessing a fully fledged synaptonemal complex, Hop1 localizes to meiotic chromosome axes, called linear elements, in fission yeast (Lorenz et al. 2004). However, the deletion of hopl has little impact on linear element formation per se (Lorenz et al. 2006). We were keen to understand whether the presence of the HORMA domain is required for localization of Hop1 to linear elements. We used immunostaining on chromatin spreads from meiotic fission yeast cells to detect heterozygously 13 myc-tagged Hop1-sv1, Hop-sv2, and Hop1- $\triangle$ HORMAD in homozygous hopl-sv1, hop1-sv2, and hopl- $\triangle H O R M A D$ strains, respectively. Hop1-sv1-13myc formed linear elements, and all four morphological classes of linear elements (dots, threads, networks, and bundles) were observed (Fig. 3a-d) (Bähler et al. 1993; Lorenz et al. 2004, 2006). This result is consistent with the wild-type phenotype for meiotic recombination of hop1-sv1 crosses. Intriguingly, Hop1-sv2-13myc was indistinguishable from Hop1-sv1-13myc (Fig. 3e-h). Therefore, the loss of the 7 AAs close to the boundary between the 


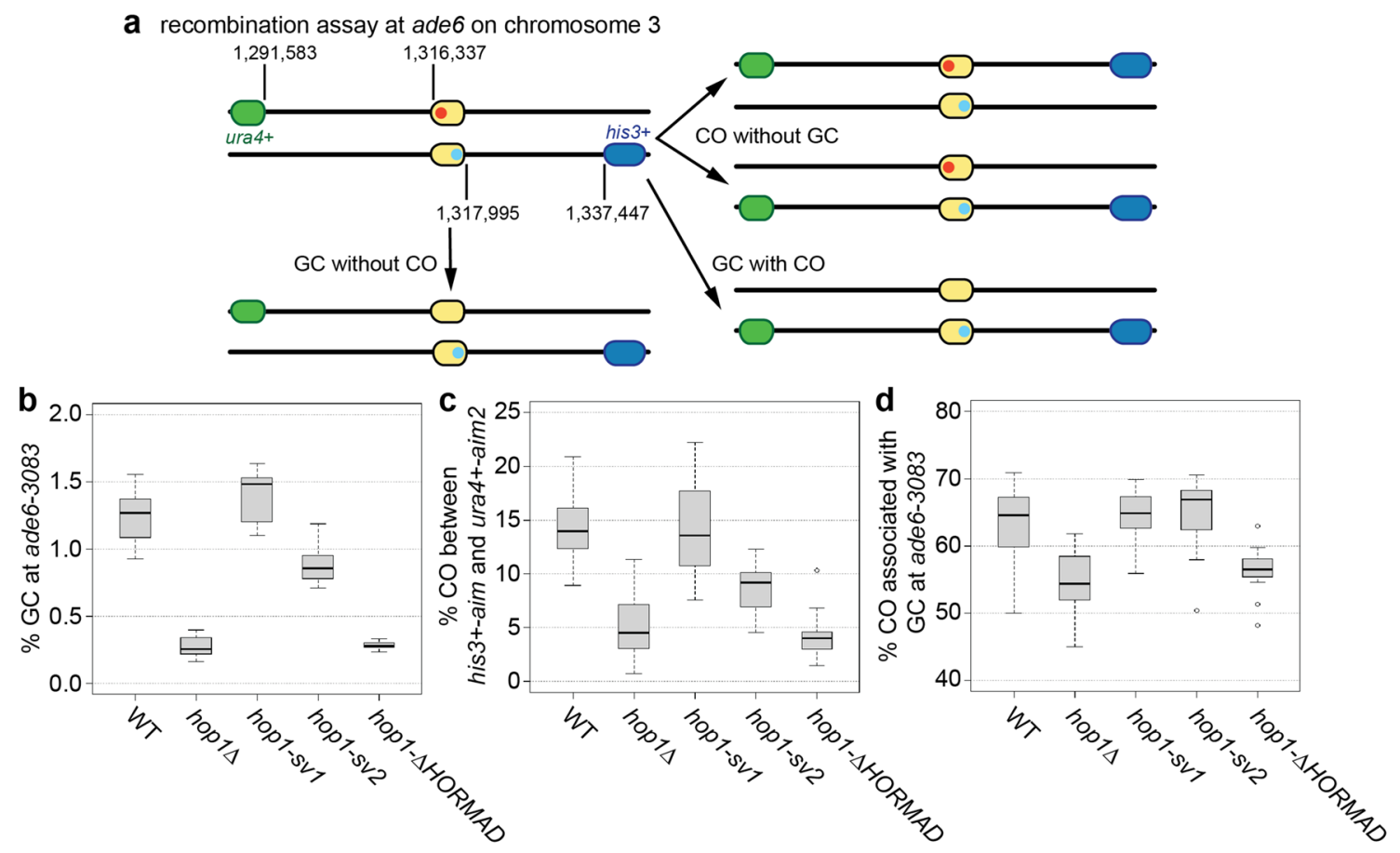

Fig. 2 Wild-type and hopl mutant levels of meiotic recombination at the ade6-3083 hotspot. a Schematic of the recombination assay at ade6 (yellow) and its prevalent outcomes: positions of ade6 and the artificially introduced markers $\mathrm{ura4}^{+}$-aim2 (green) and his $3^{+}$aim (blue) on chromosome III are given in bp; positions of the point mutations in ade6 in the two parental strains are indicated in red (ade6-3083) and light blue (ade6-469). b Frequency of gene conversion (GC) at ade6-3083, c frequency of crossovers (COs) between

HORMA core domain and the HORMA "safety-belt" region in the hop1-sv2 allele does not conspicuously influence recruitment of Hop1 to linear elements, despite affecting meiotic recombination (Fig. 2b-d). Hop1- $\triangle$ HORMAD13 myc could not be detected on meiotic chromatin (Fig. S4) [a GFP-tagged version of the linear element protein Mug20 served as control to identify meiotic nuclei containing linear elements (Estreicher et al. 2012)], which is consistent with hop 1- $\triangle H O R M A D$ displaying a meiotic recombination phenotype similar to hop $1 \Delta$ (Fig. 2b-d).

\section{Searching for Hop1 interactors}

A bio-informatics screen using the Pombe Interactome (PInt) (Pancaldi et al. 2012) for potential Hop1 interactors retrieved SPAC688.03c as the highest hit from an uncharacterized open reading frame (10th highest overall). The gene product of SPAC688.03c localizes preferentially to the nucleus (Matsuyama et al. 2006) (Fig. S5) and is upregulated at the onset of meiosis (Mata et al. 2002), which is consistent with a potential role in meiotic chromatin metabolism. Notably, a point mutation in the human ortholog of SPAC688.03c, $A M M E C R 1$, is associated with Alport syndrome (A), mental $\mathrm{ura}^{+}$-aim 2 and $h i s 3^{+}$-aim, and $\mathbf{d}$ percentage of COs between ura $^{+}$-aim 2 and his $3^{+}$-aim associated with a GC event at ade6-3083 in crosses of wild type (WT) ALP733 $\times$ ALP731 $(n=12)$, hopl $\Delta$ UoA200 $\times \operatorname{UoA} 199(n=17)$, hopl-sv1 UoA766 $\times \operatorname{UoA767}(n=10)$, hop1-sv2 UoA853 $\times$ UoA854 $(n=12)$, and hopl- $\triangle H O R M A D$ UoA945 $\times \operatorname{UoA} 944(n=12) ; n$ indicates the number of independent crosses (see also Table S2)

retardation $(\mathrm{M})$, midface hypoplasia $(\mathrm{M})$, and elliptocytosis (E) (Vitelli et al. 1999; Andreoletti et al. 2017). Therefore, we investigated the phenotype of a SPAC688.03c (from here on ahol, for AMMECRl-homolog) deletion in vegetative and generative cells.

First, ahol $\Delta$ was assayed for changes in genotoxin sensitivity and meiotic recombination outcome. The wild-type and ahol $\Delta$ strains exhibited similar levels of resistance to the topoisomerase I-poison camptothecin (CPT), the alkylating agent methyl methanesulfonate (MMS), the ribonucleotide reductase blocker hydroxyurea (HU), and ultraviolet (UV) radiation (Fig. 4a). The deletion of ahol in a wildtype or hopl $\Delta$ background did not affect spore viability (Fig. 4b). The meiotic recombination outcome of an ahol $\Delta$ single mutant was similar to wild type, and an ahol $\Delta$ hopl $\Delta$ double mutant was indistinguishable from a hopl $\Delta$ single mutant, except for a moderate 1.4-fold reduction in gene conversion ( $p=0.034$ ) (Fig. 4c-e, Table S2). These results indicate that under standard laboratory conditions $a h o l^{+}$is not required for DNA damage repair or meiotic recombination. Therefore, Aho1 is not an essential interactor of Hop1 because it does not mirror the phenotype of hopl $\Delta$ and we did not observe strong genetic interactions with hopl $\Delta$. 

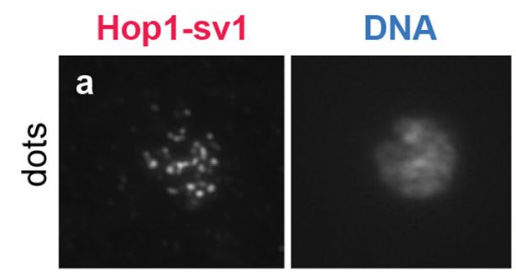

merge
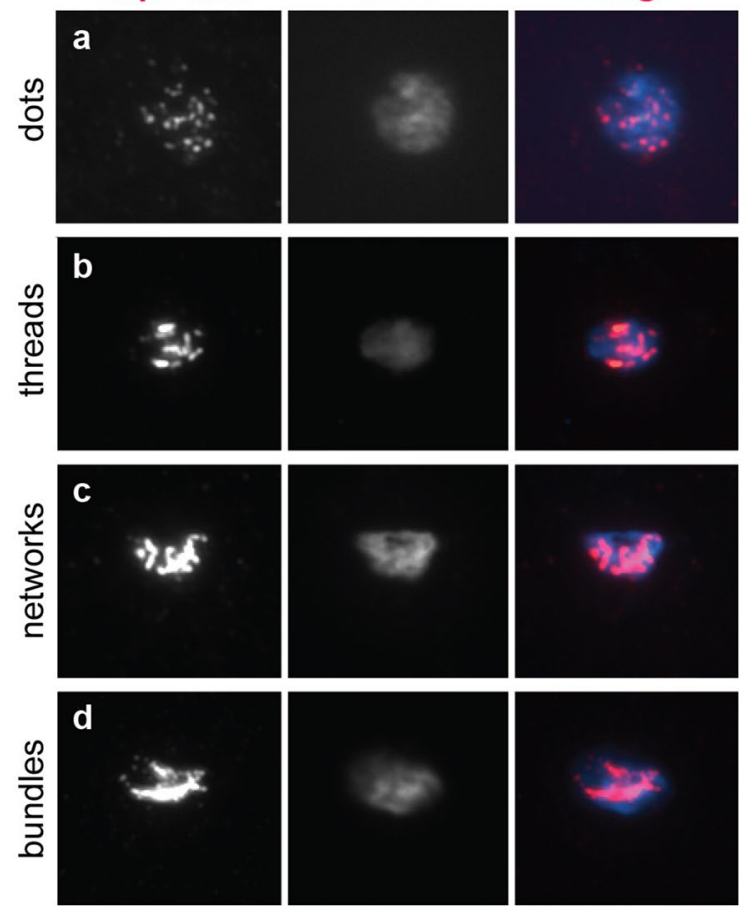

Fig. 3 Linear element morphology delineated by immunostaining of Hop1-13myc on chromatin spreads from meiotic fission yeast cells. Linear elements as seen by immunostaining against Hop1-13myc develop from dots via threads to networks and bundles in $h^{+N} / h^{-s m t 0} h o p 1$ sv1/hop1-sv1::13myc-kanMX6 ade6-M210/ade6-M216 (UoA878) (a-d)

We tested whether overexpression of $a \mathrm{hol}^{+}$might uncover a DNA repair and/or recombination role. The $a h o 1^{+}$ gene was put under the control of the thiamine-repressible nmt 1-promoter at the medium pREP41-level and tagged with GFP at its $\mathrm{C}$ terminus. Spot assays and recombination assays were performed after Aho1-GFP over-expression was induced for $24 \mathrm{~h}$ (see also Fig. S5). Cells over-expressing Aho1-GFP did not show increased genotoxin sensitivities compared to cells containing an empty plasmid, cells overexpressing GFP alone, or cells grown in the presence of thiamine (in which expression is not induced) (Fig. 5a, b). Over-expression of Aho1-GFP also did not alter spore viability and meiotic recombination outcome in comparison to GFP overexpression (Fig. 5c-f, Table S3), indicating that a massive surplus of Aho1 protein has no negative impact on reproductive success.

\section{hop1 displays genetic interactions with the structure-selective endonuclease mus 81 and the DNA helicase $\mathrm{fm} / 1$}

Considering that the absence of hopl reduces recombination outcome in a similar fashion to Rad51/Dmc1-mediator (rad55-rad57, swi5-sfrl, and rlp1-rdl1-sws1) mutants
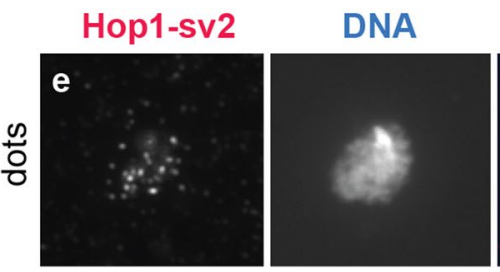

merge
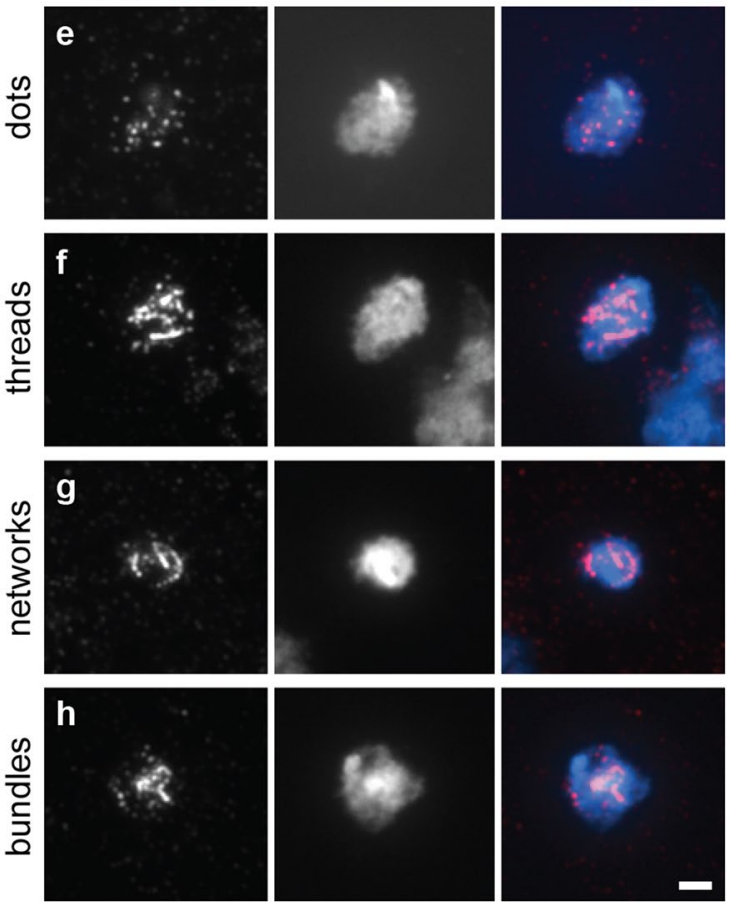

or $h^{+N} / h^{-s m t 0}$ hop1-sv2/hop1-sv2::13myc-kanMX6 ade6-M210/ade6M216 (UoA930) (e-h). Hop1-13myc is shown in red and Hoechst 33342-stained DNA in blue in the merge panels. Scale bar represents 2 $\mu \mathrm{m}$

(Lorenz et al. 2012, 2014) (Fig. 2) we were wondering how hopl genetically interacts with the key meiotic determinants of $\mathrm{CO}$ and non-CO formation, mus81 and fmll.

The deletion of hop $1 \Delta$ in a mus $81 \Delta$ background improves spore viability from 1.85 to $18.7 \%$ (Fig. 6, Table S2) similar to what has been seen in mus81 $\Delta$ Rad51/Dmc1-mediator double mutants (Lorenz et al. 2010, 2012, 2014). This rescue in spore viability is not accompanied by a restoration of $\mathrm{CO}$ frequency. A hopl $\Delta$ mus $81 \Delta$ mutant shows only $0.2 \%$ of COs which is significantly different both from wild type $\left(p=1.9 \times 10^{-7}\right)$ and the hop $1 \Delta$ single mutant $\left(p=2.7 \times 10^{-3}\right)$ (Fig. 6c, Table S2). Also, the rate of COs associated with GC events at $0.71 \%$ is significantly lower in the hop $1 \Delta$ mus $81 \Delta$ double mutant than in the wild type $\left(63.0 \%, p=5.3 \times 10^{-12}\right)$ or the hopl $\Delta$ single mutant $\left(54.3 \%, p=3.4 \times 10^{-15}\right)$ (Fig. 6d, Table S2).

Removal of $\mathrm{fmll}^{+}$increases COs associated with GC events to $72.2 \%$ ( $\sim 10$ percentage points more than wild type) (Lorenz et al. 2012), but has little effect on other recombination outcomes (Fig. 6). In fmll $\Delta$ hop $1 \Delta$ double mutants, there are $72.6 \%$ of COs associated with GC events which is significantly different from both wild type $\left(p=3.4 \times 10^{-3}\right)$ and a hop $1 \Delta$ single mutant $\left(p=4.9 \times 10^{-9}\right)$, but indistinguishable from a fmll $\Delta$ single mutant $(p=0.78)$ (Fig. 6d, 

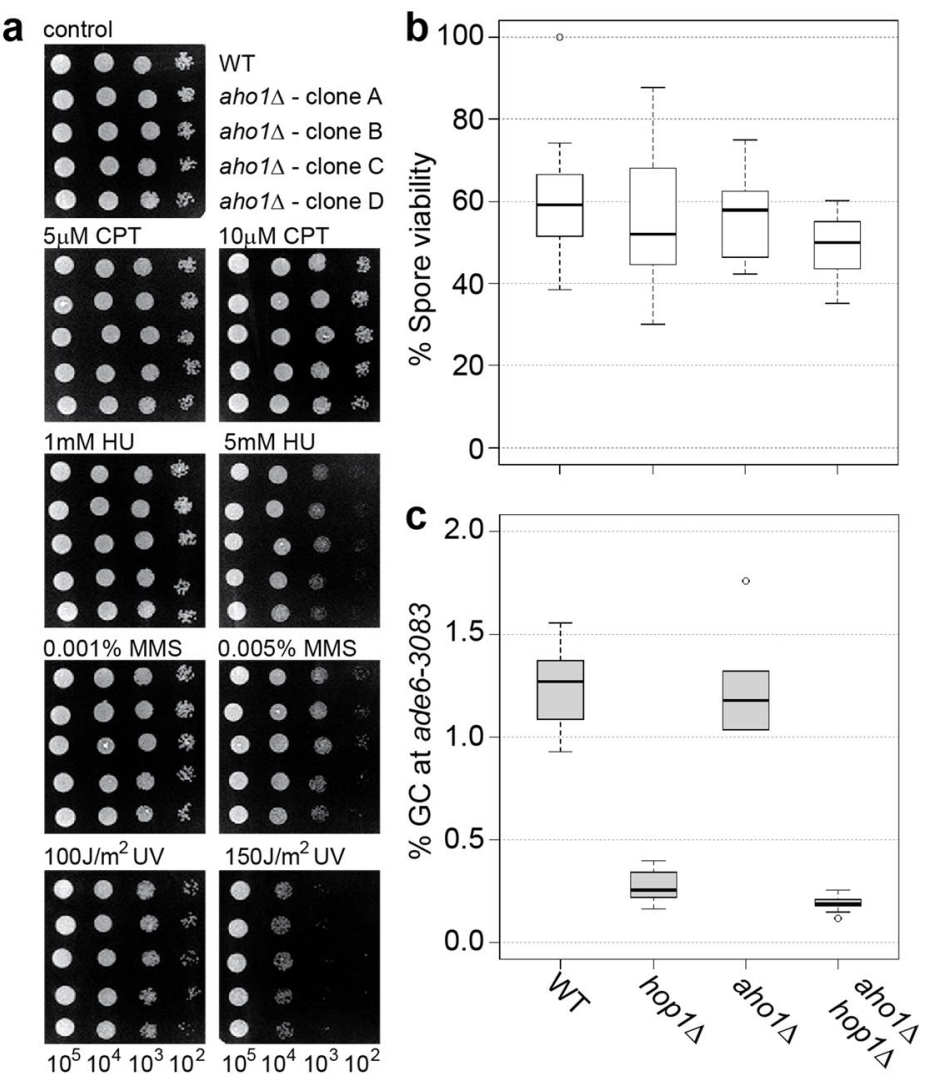
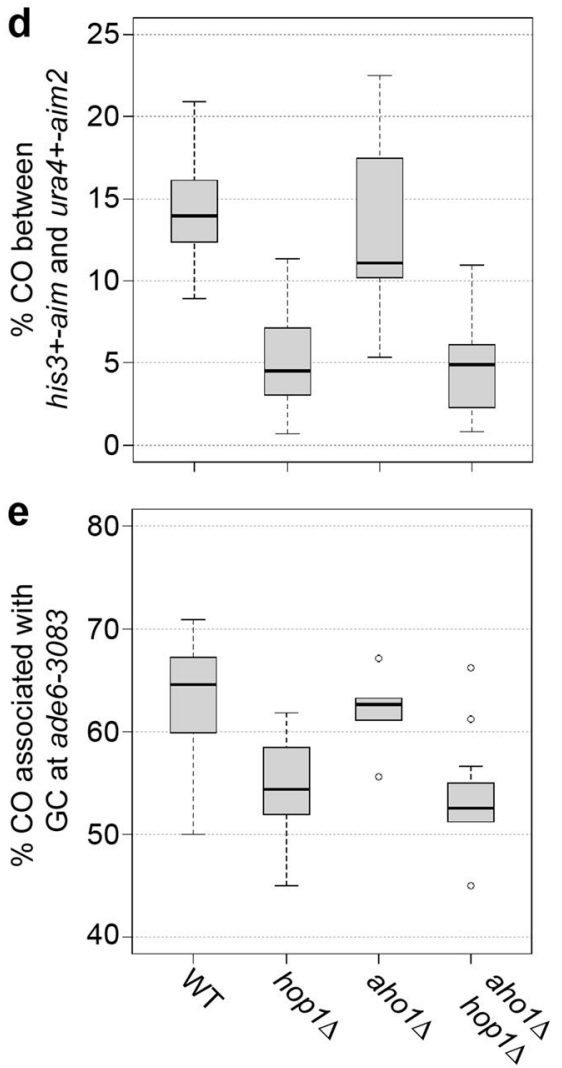

Fig. 4 The role of $a h o I^{+}$in genotoxic stress resistance and meiotic recombination. a Spot assays comparing wild type (WT, FO652) and ahol $\Delta$ (UoA423) for sensitivity to a range of genotoxins; number of cells plated in each spot is indicated at the bottom of the figure. b Percent spore viability, c frequency of gene conversion (GC) at ade6-3083, d frequency of crossovers (COs) between ura $^{+}$-aim2 and his $3^{+}$-aim, and e percentage of COs between $u r a 4^{+}$-aim 2 and his $3^{+}$-aim associated with a GC event at ade6-3083 in crosses of wild type (WT) ALP733 $\times$ ALP731 $(n=12)$, hopl $\Delta$ UoA200 $\times$ UoA199

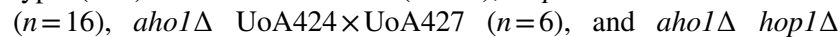
UoA785 $\times \operatorname{UoA786}(n=13) ; n$ indicates the number of independent crosses (see also Table S2)
Table S2). Overall CO formation is not changed compared to hopl $\Delta(p=0.78)$, but significantly different from wild type $\left(p=7 \times 10^{-7}\right)$ and fmll $\Delta\left(p=6.3 \times 10^{-8}\right)$ (Fig. $6 \mathrm{c}$, Table S2). Also GC frequency is unchanged compared to hopl $\Delta(p=0.49)$ (Fig. 6b, Table S2) suggesting that in the absence of hop $1^{+}$the DNA helicase Fml1 does not gain any additional functionality.

\section{hop1 does not show strong genetic interactions with Rad51/Dmc1-mediator mutants}

Rad51/Dmc1-mediators are highly conserved, positive determinants of meiotic recombination (Schwacha and Kleckner 1997; Ellermeier et al. 2004; Hayase et al. 2004; Bleuyard et al. 2005; Hyppa and Smith 2010; Lorenz et al. 2012, 2014; Sasanuma et al. 2013). In fission yeast the meiotic recombination phenotype of the hop $1 \Delta$ crosses strongly resembles that of Rad51/Dmc1-mediator mutants in that it reduces all meiotic recombination outcomes and alleviates the effects of a mus $81 \Delta$ mutants (see above). Therefore, we were interested in how hopl genetically interacts with the mediators $s f r l$, rad55, and $r l p l$, as well as $d m c l$ itself. The deletion of hopl ${ }^{+}$leads to a notable reduction in DSB formation (Rothenberg et al. 2009; Latypov et al. 2010), and some of the decrease in recombination frequency in hopl $\Delta$ is likely due to low DSB formation. This reduction may be unrelated to Rad51/Dmc1-mediator function, because absence of the Sfr1-cofactor $\mathrm{swi}^{+}$or of $d m c \mathrm{I}^{+}$does not appreciably reduce DSB formation (Young et al. 2004). We pose that in the absence of $\mathrm{hopl}^{+}$, which contributes to the establishment of a meiosis-specific chromatin environment, successful sexual reproduction will become more reliant on the mitotic repair factors Rad55 and Rlp1. Therefore, we expected a substantial negative genetic interaction of hopl with $s f r l$, rad55, $r l p l$, and $d m c l$.

Indeed, hop $1 \Delta \operatorname{rad} 55 \Delta$ and hopl $\Delta \operatorname{rlpl} \Delta$ double mutants displayed significantly lower GC $\left(p=5.3 \times 10^{-5}\right.$ and $p=0.02$, respectively) than a hop $1 \Delta$ single mutant; the GC level is also notably lower than in rad55 $\Delta$ $\left(p=2.9 \times 10^{-5}\right)$ and $\operatorname{rlpl} \Delta\left(p=1.3 \times 10^{-4}\right)($ Fig. $7 \mathrm{a}$, 

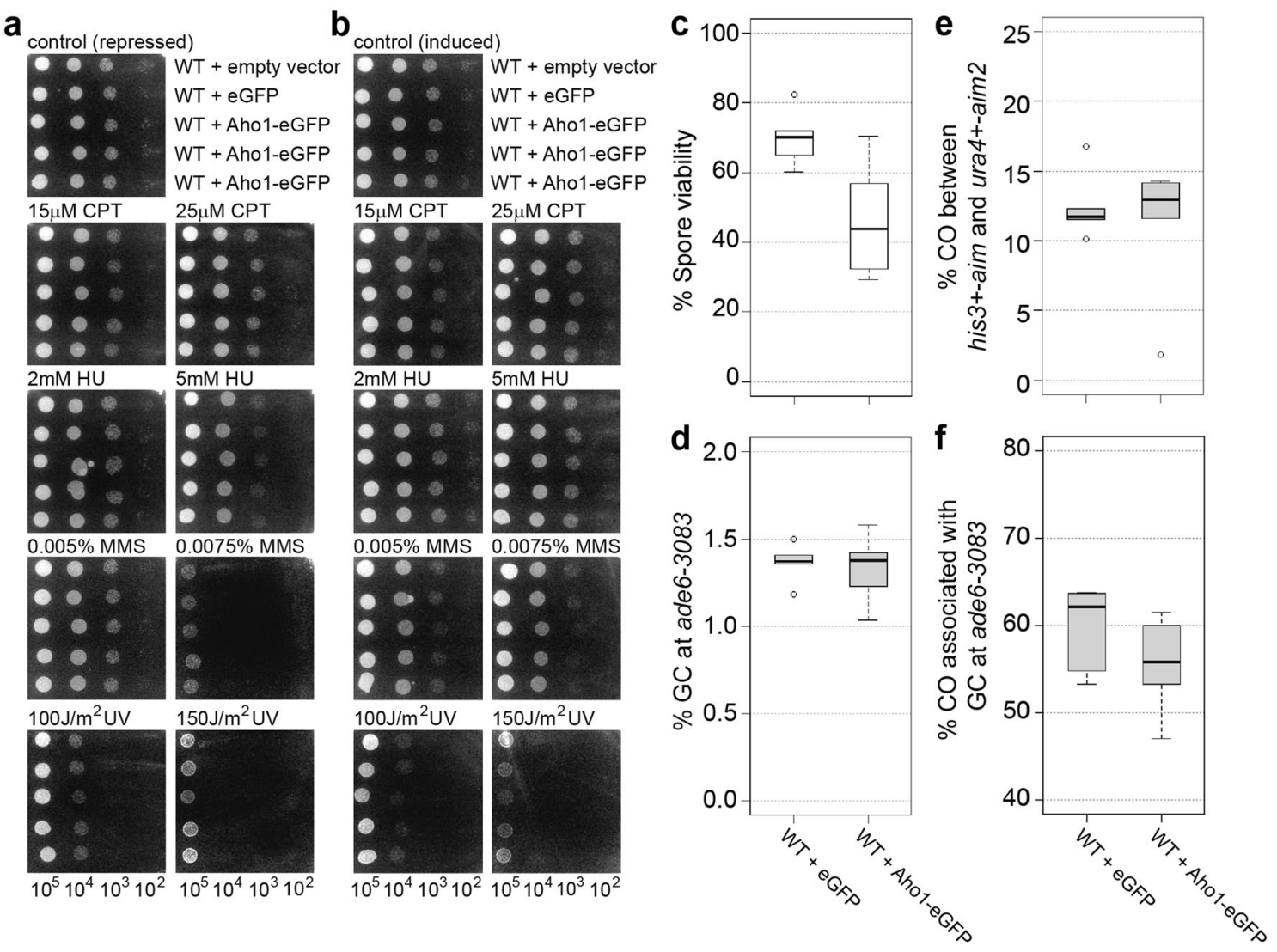

Fig. 5 Overexpression of the $a h o l^{+}$in wild-type fission yeast cells and its consequences for genotoxic stress resistance and meiotic recombination. a, b Effect of GFP and Aho1-GFP overexpression on the genotoxin sensitivity of wild type (WT, FO652); number of cells plated in each spot is indicated at the bottom of the figure. $\mathbf{c}$ Percent spore viability, d frequency of gene conversion (GC) at ade6-3083,

Table S2) (Lorenz et al. 2014). However, overall CO frequency and COs associated with GC events were not significantly different from hop $1 \Delta$ or rad55 $\Delta$ in hopl $\Delta \operatorname{rad} 55 \Delta$, and from hopl $\Delta$ or rlpl $\Delta$ in hopl $\Delta$ rlpl $\Delta$ (Fig. 7b, c; Table S2). Deletion of $s f r 1^{+}$decreased GC rate by 7.5 -fold in a hop $1 \Delta$ background to $0.036 \%\left(p=1.4 \times 10^{-8}\right)$, which is also significantly lower than the $0.13 \%\left(p=1 \times 10^{-4}\right)$ found in an $s$ fr $1 \Delta$ single mutant (Fig. 7d, Table S2) (Lorenz et al. 2014). Other recombination outcomes were indistinguishable from hopl $\Delta$ (Fig. 7e, f; Table S2). The interaction of hopl $\Delta$ with $d m c l \Delta$ is completely epistatic (Fig. 7d-f, Table S2). Importantly, no noteworthy decreases of spore viability have been observed in any of the double mutants (Table S2). This stands in contrast to double mutant combinations of Rad51/Dmc1-mediators with each other or with $d m c 1 \Delta ; d m c 1 \Delta \operatorname{rad} 55 \Delta, d m c 1 \Delta$ $\operatorname{rlpl} 1 \Delta, \operatorname{rad} 55 \Delta$ sfr $1 \Delta$ and $r l p l \Delta$ sfrl $\Delta$ display strong synergistic reductions in spore viability (Lorenz et al. 2014). Overall, this suggests that in fission yeast meiosis the absence of $h_{o p I^{+}}$does not generate a situation which

e frequency of crossovers (COs) between $\mathrm{ura}^{+}$-aim 2 and $\mathrm{his} 3^{+}$-aim, and $\mathbf{f}$ percentage of COs between $\mathrm{ura}^{+}$-aim 2 and his $3^{+}$-aim associated with a GC event at ade6-3083 in crosses of wild type (WT) ALP733 $\times$ UoA841 overexpressing GFP $(n=6)$, or Aho1-GFP $(n=6)$; $n$ indicates the number of independent crosses (see also Table S3)

necessitates the presence of a fully functional recombination machinery to produce viable progeny.

\section{Discussion}

Here, we characterize the genetic interactions of hopl, which encodes a meiotic HORMA domain protein, with homologous recombination factors in fission yeast. Meiotic HORMADs are components of the meiosis-specific chromosome axis which promote DSB formation to allow for the efficient pairing of homologous chromosomes. At the same time HORMADs attenuate undesirable recombination between sister chromatids (reviewed in Rosenberg and Corbett 2015). In organisms possessing a synaptonemal complex, HORMADs support its formation, and depending on the organism may mount a checkpoint arrest when DSB repair is not completed or homologous chromosomes are not synapsed (Woltering et al. 2000; Armstrong et al. 2002; Couteau and Zetka 2005; Martinez-Perez and Villeneuve 

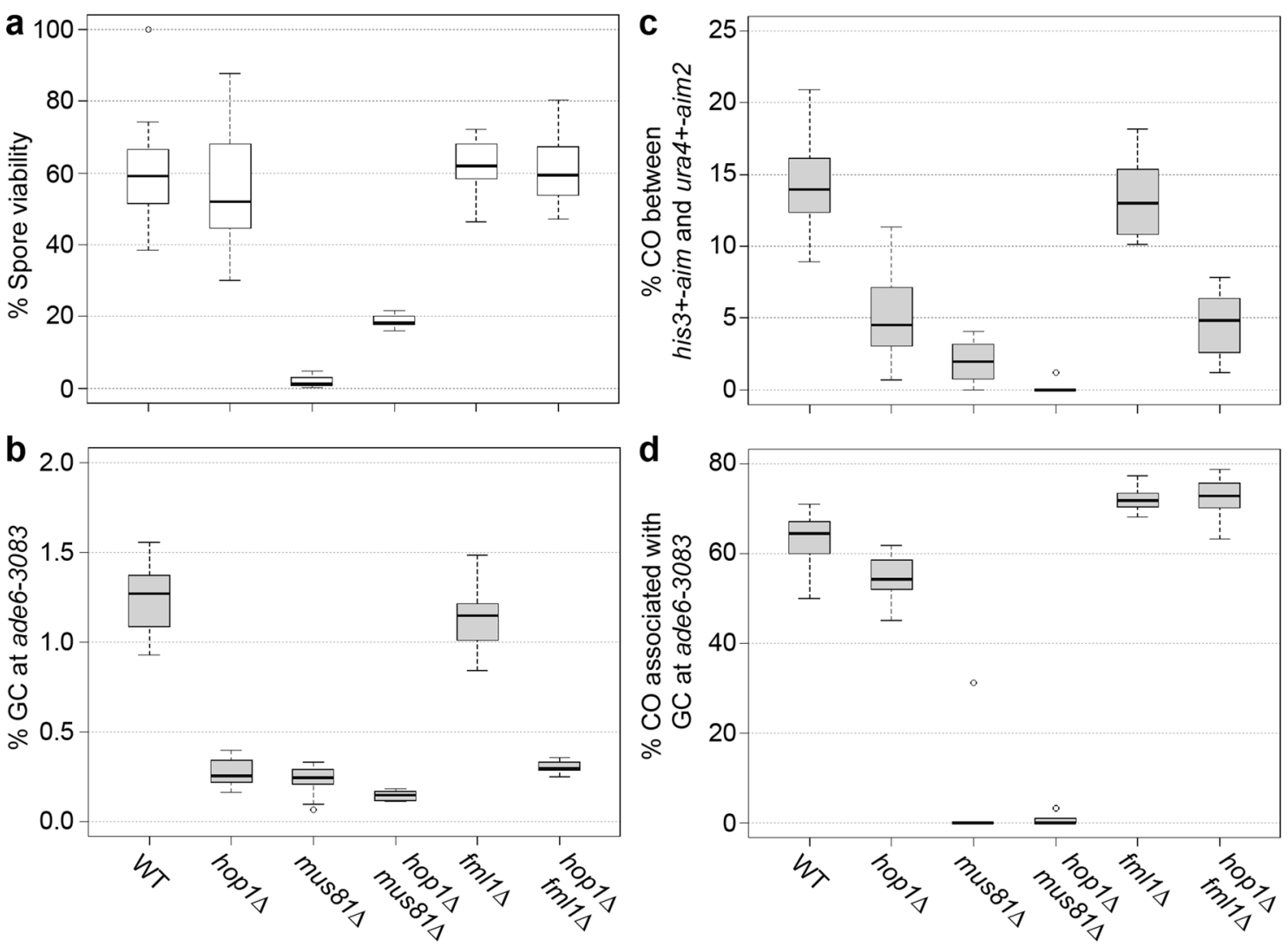

Fig. 6 Genetic interaction of hop $1 \Delta$ with deletions of factors required for meiotic recombination intermediate processing. a Percent spore viability, b frequency of gene conversion (GC) at ade6-3083, c frequency of crossovers (COs) between ura $^{+}$-aim 2 and his $3^{+}$-aim, and $\mathbf{d}$ percentage of COs between $\mathrm{ura}^{+}$-aim 2 and his $3^{+}$-aim associated with a GC event at ade6-3083 in crosses of wild type (WT)

2005; Carballo et al. 2008; Daniel et al. 2011; Rinaldi et al. 2017). Sz. pombe meiosis progresses without the formation of a synaptonemal complex; therefore, any roles related to the synaptonemal complex will likely not apply. Indeed, DSB formation is reduced and inter-sister recombination is increased in fission yeast hopl $\Delta$ (Rothenberg et al. 2009; Latypov et al. 2010). Consistent with DSB reduction, decreased numbers of Rec7-foci (an essential co-factor of Spo11) have been observed when hopl was absent (Lorenz et al. 2006). As a result of DSB reduction, meiotic interhomolog recombination frequencies are lower in hopl $\Delta$ than in wild type (Latypov et al. 2010) (Fig. 2). Thus, the Hop1 function in promoting DSB formation seems to be conserved in fission yeast. In budding yeast Hop1 also acts downstream of DSB formation in promoting Dmc1-dependent interhomolog recombination by preventing Dmc1-independent Rad51-driven recombination (Niu et al. 2005; Carballo et al. 2008). The suppression of inter-sister recombination is achieved by Hop1-dependent activation of Mek1 which in turn phosphorylates and deactivates the Rad51-mediator

ALP733 $\times$ ALP731 $(n=12)$, hop1 $\Delta$ UoA200 $\times$ UoA199 $(n=16)$, mus81 $\triangle$ ALP812 $\times$ ALP813 $(n=10$; in a) and ALP802 $\times$ ALP822 $(n=10$; in c, d), hop1 $\Delta$ mus81 $\Delta$ UoA204 $\times \operatorname{UoA} 203(n=6)$, fmll $\Delta$ ALP1133 $\times$ FO2608 $(n=15)$, and fmll $\Delta$ hop1 $\Delta$ UoA182 $\times$ UoA181 $(n=14) ; n$ indicates the number of independent crosses (see also Table S2)

Rad54, and also phosphorylates and activates the meiosisspecific Rad51-inhibitor Hed1 (Tsubouchi and Roeder 2006; Niu et al. 2009; Callender et al. 2016). Together, this regulatory network has specific genetic consequences: (1) meiotic recombination cannot proceed without Dmc1 which causes a meiotic arrest resulting in an inability to sporulate, and (2) deleting hopl in a $d m c 1 \Delta$ background restores spore formation by allowing Rad51-dependent DSB repair (Niu et al. 2005; Carballo et al. 2008). In fission yeast the genetic interaction between hopl and $d m c l$ looks quite different (Fig. 7) in that it is epistatic, however neither single mutant has as strong a phenotype as the corresponding mutants in S. cerevisiae (Hollingsworth and Byers 1989; Bishop et al. 1992; Grishchuk and Kohli 2003; Latypov et al. 2010). Two features may account for this apparent difference between the two yeasts: the budding yeast is probably the only organism which shuts down Rad51 strand exchange activity via Hed1 (Tsubouchi and Roeder 2006), and fission yeast employs both Rad51 and Dmc1 in their direct strand exchange capacity (Grishchuk and Kohli 2003). Interestingly, the genetic 

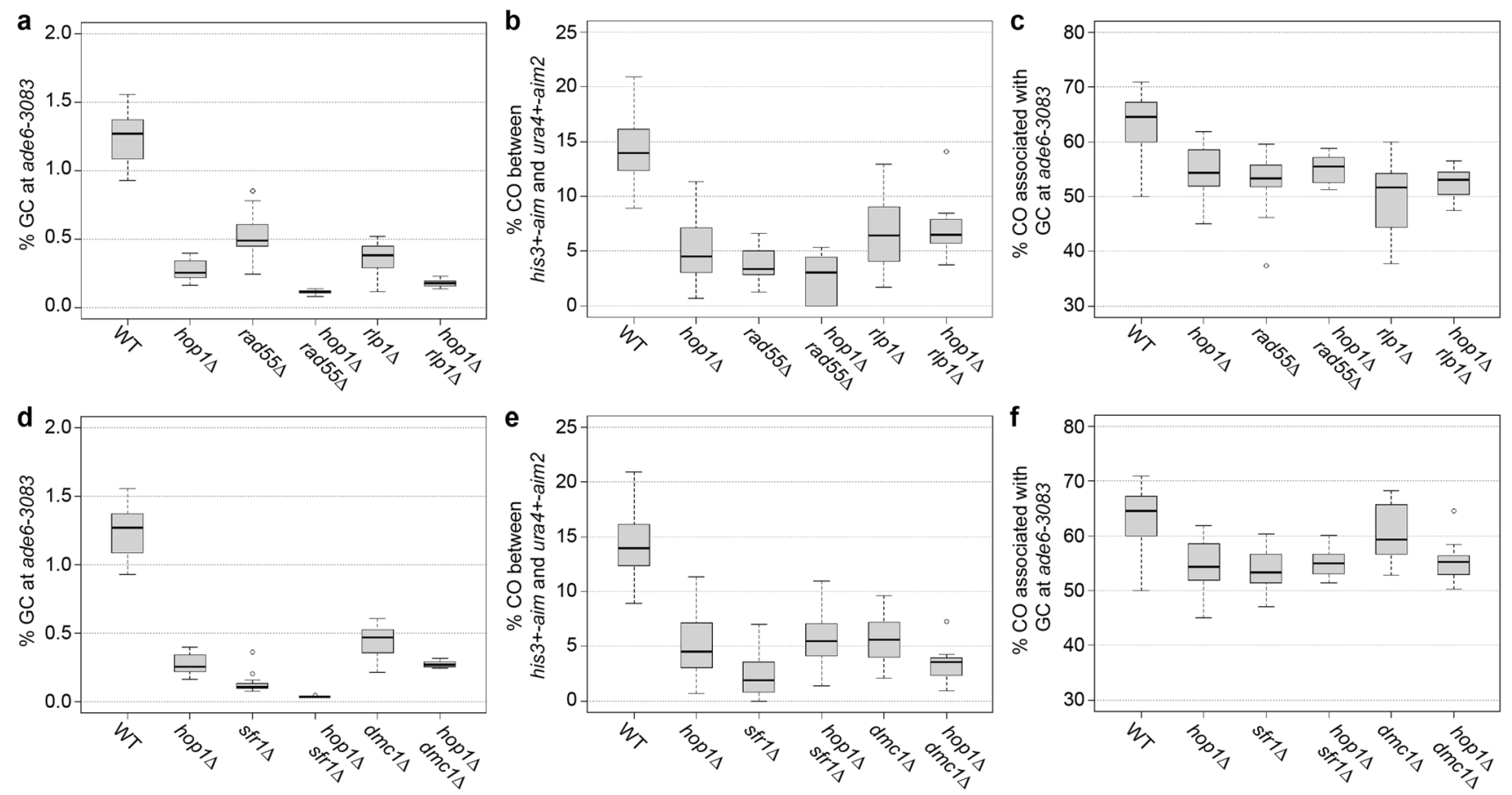

Fig. 7 Genetic interaction of hop $1 \Delta$ with deletions of Rad51/Dmc1mediator genes. a, d Frequency of gene conversion (GC) at ade63083, b, e frequency of crossovers (COs) between $u r a 4^{+}$-aim 2 and his $3^{+}$-aim, and c, f percentage of COs between $u r a 4^{+}$-aim 2 and his $3^{+}$-aim associated with a GC event at ade6-3083 in crosses of wild type (WT) ALP733 $\times$ ALP731 $(n=12)$, hop1 $\Delta$ UoA200 $\times$ UoA199

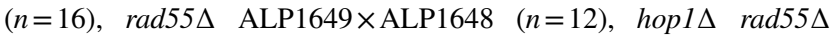

interaction of meiotic Hormad and $d m c 1$ genes generally does not show much conservation. In Arabidopsis mutation of asyl or $d m c l$ causes a strong reduction or absence of chiasmata, respectively, leading to fertility defects. However, the asyl $d m c 1$ double mutant looks like a $d m c l$ single mutant (Sanchez-Moran et al. 2007). In the mouse both $\mathrm{Dmcl}^{-/-}$and Hormadl ${ }^{-/-}$single mutants undergo meiotic arrest at mid-pachytene, and so does the $\mathrm{Dmcl}^{-1-} \mathrm{Hor}$ mad1 ${ }^{-1-}$ double mutant (Daniel et al. 2011), i.e., there is no reciprocal rescue as in $S$. cerevisiae hop $1 \Delta d m c 1 \Delta$. These disparate observations in various model organisms suggest that the genetic networks controlling DSB repair and CO formation, and how these processes are integrated with chromosome axis organization, have undergone considerable rewiring during evolution. Because fission yeast relies on both Rad51 and Dmc1 to invade a repair template during meiotic DSB repair, we also looked at the genetic interaction of hopl with Rad51/Dmc1-mediator mutants. Apart from an additive effect on GC frequency in hopl $\Delta$ rad55 $\Delta$, hop $1 \Delta$ $r l p l \Delta$, and hop $1 \Delta$ sfrl $1 \Delta$ double mutants, which can easily be explained by the early functions of Hop1 (reduced DSB formation and increased inter-sister recombination), other recombination outcomes were not different from the hop $1 \Delta$ single mutant (Fig. 7). This indicates that in a fission yeast
UoA846 $\times$ UoA847 $(n=10), \quad r l p 1 \Delta$ ALP1623 $\times$ ALP1620 $(n=18)$, hopl $\Delta$ rlpl $\Delta$ UoA848 $\times$ UoA849 $(n=10)$, sfrl $\Delta$ ALP800 $\times$ ALP782 $(n=22), \quad h o p 1 \Delta s f r 1 \Delta \quad \mathrm{UoA} 844 \times \mathrm{UoA} 845 \quad(n=12), \quad d m c 1 \Delta$ ALP1545 $\times \operatorname{ALP} 1544(n=12)$, and $d m c 1 \Delta h o p 1 \Delta \mathrm{UoA} 851 \times \mathrm{UoA} 852$ $(n=10) ; n$ indicates the number of independent crosses (see also Table S2)

hopl $\Delta$ mutant gamete production does not become more reliant on strand exchange than in a wild-type background.

Genetic interaction of hopl $1 \Delta$ with mus $81 \Delta$ resembled that of mus $81 \Delta$ combined with mutants affecting strand exchange (Ellermeier et al. 2004; Lorenz et al. 2012, 2014), rescuing mus $81 \Delta$ 's spore viability defect without rescuing its $\mathrm{CO}$ defect (Fig. 6). This interaction can be explained by Hop1 promoting DSB formation and inter-homolog recombination: fewer DSBs and fewer inter-homolog events in hop $1 \Delta$ decrease the probability of the production of recombination intermediates requiring the action of Mus81. Additionally, the absence of hopl might allow DNA helicases to process recombination intermediates as non-COs more efficiently than in a wild-type background.

It is well established that fungal Hop1 localizes to the chromosome axis during meiotic prophase by physically interacting with the linear/lateral element protein Rec10/ Red1 in fission and budding yeast, respectively (de los Santos and Hollingsworth 1999; Spirek et al. 2010). Considering Hop1's central role in coordinating meiotic progression, it is not unlikely that it would physically interact with previously uncharacterized meiotic players; indeed with IHO1, a novel HORMAD1-interactor has recently been described in mice (Stanzione et al. 2016). A bio-informatical protein 
interaction-predictor PInt (Pancaldi et al. 2012) suggested that the uncharacterized AMMECR1-homolog Aho1 might be a potential Hop1-interactor in fission yeast. However, neither deletion nor over-expression of Aho1 indicates a clear meiotic function, independent of the presence or absence of Hop1 (Figs. 4, 5).

One of the determining features of Hop1 is its HORMA domain whose function was enigmatic until recently, when it was implicated in the recruitment of Hop 1 to the meiotic chromosome axis and in its self-assembly into larger homomeric complexes (Kim et al. 2014; Rosenberg and Corbett 2015). We exploited a cDNA clone of hopl (hopl-sv2) which produces a protein that lacks 7 AAs in the HORMA domain and constructed a version of hop1 lacking the complete HORMA domain (hopl- $\mathrm{HORMAD)}$ to examine whether the HORMA domain in fission yeast Hop1 is essential for its function and localization. Interestingly, expressing Hop1-sv2 in place of wild-type Hop1 did not result in a notable reduction in localization to chromatin, however it did cause a hypomorphic phenotype for GC and CO frequency outcome, whereas the complete removal of the HORMA domain from Hop 1 mimicked a deletion phenotype (Figs. 2, 3 , S4). This suggests that the shortened HORMA domain in Hop1-sv2 is still partially functional, because absence of Hop1's HORMA domain in fission yeast completely abolishes localization to meiotic chromatin causing a hopl $\Delta$ phenotype in recombination outcome.

It is very interesting how genetic pathways driving meiotic chromosome organization and meiotic recombination are rewired during evolution. The fission yeast, which is lacking a synaptonemal complex, but retains remnants of a meiosis-specific chromosome axis, is a great model for probing the roles of conserved meiotic factors such as Hop1. Clearly, Hop1 fulfills an important meiotic function in promoting DSB formation and inter-homolog recombination in this organism, but lacks regulatory roles in lieu of the synaptonemal complex, which appears crucial in the majority of organisms performing meiosis with a synaptonemal complex.

Acknowledgements We are grateful to Shin-ichiro Hiraga, Franz Klein, Jürg Kohli, Josef Loidl, Walter W. Steiner, Matthew C. Whitby, and the National BioResource Project (NBRP) Japan for providing strains and/or plasmids, and to K.C. Chan, M. Roca, and D. Whyte for technical assistance. Kevin Corbett, Nancy Hollingsworth, Takashi Kubota, and Josef Loidl are thanked for critically reading a previous version of this manuscript. Microscopy was performed at the University of Aberdeen Microscopy and Histology facility (Kevin Mackenzie). This work was supported by the Biotechnology and Biological Sciences Research Council UK (BBSRC, Doctoral Training Grant BB/ F016964/1), and the University of Aberdeen (College of Life Sciences and Medicine Start-up Grant to AL).

Author contributions SDB: unpublishing of essential reagents (yeast strains, plasmids), acquisition of data, analysis and interpretation of data, revising the manuscript; ODJ: acquisition of data, revising the manuscript; AL: conception and design, unpublishing of essential reagents (yeast strains, plasmids), acquisition of data, analysis and interpretation of data, drafting and revising the manuscript.

Open Access This article is distributed under the terms of the Creative Commons Attribution 4.0 International License (http://creativeco mmons.org/licenses/by/4.0/), which permits unrestricted use, distribution, and reproduction in any medium, provided you give appropriate credit to the original author(s) and the source, provide a link to the Creative Commons license, and indicate if changes were made.

\section{References}

Akamatsu Y, Dziadkowiec D, Ikeguchi M et al (2003) Two different Swi5-containing protein complexes are involved in matingtype switching and recombination repair in fission yeast. Proc Natl Acad Sci USA 100:15770-15775. https://doi.org/10.1073/ pnas. 2632890100

Andreoletti G, Seaby EG, Dewing JM et al (2017) AMMECR1: a single point mutation causes developmental delay, midface hypoplasia and elliptocytosis. J Med Genet 54:269-277. https://doi. org/10.1136/jmedgenet-2016-104100

Aravind L, Koonin EV (1998) The HORMA domain: a common structural denominator in mitotic checkpoints, chromosome synapsis and DNA repair. Trends Biochem Sci 23:284-286. https://doi. org/10.1016/S0968-0004(98)01257-2

Armstrong SJ, Caryl AP, Jones GH, Franklin FCH (2002) Asy1, a protein required for meiotic chromosome synapsis, localizes to axis-associated chromatin in Arabidopsis and Brassica. J Cell Sci 115:3645-3655. https://doi.org/10.1242/jcs.00048

Bähler J, Wyler T, Loidl J, Kohli J (1993) Unusual nuclear structures in meiotic prophase of fission yeast: a cytological analysis. J Cell Biol 121:241-256. https://doi.org/10.1083/jcb.121.2.241

Bähler J, Wu JQ, Longtine MS, et al (1998) Heterologous modules for efficient and versatile PCR-based gene targeting in Schizosaccharomyces pombe. Yeast 14:943-951. https://doi.org/10.1002/ (SICI)1097-0061(199807)14:10<943::AID-YEA292>3.0.CO;2Y

Bishop DK, Park D, Xu L, Kleckner N (1992) DMC1: a meiosis-specific yeast homolog of E. coli $r e c A$ required for recombination, synaptonemal complex formation, and cell cycle progression. Cell 69:439-456. https://doi.org/10.1016/0092-8674(92)90446-J

Bleuyard J-Y, Gallego ME, Savigny F, White CI (2005) Differing requirements for the Arabidopsis Rad51 paralogs in meiosis and DNA repair. Plant J 41:533-545. https://doi.org/10.1111/j.1365313X.2004.02318.x

Brown MS, Bishop DK (2014) DNA strand exchange and RecA homologs. Cold Spring Harb Perspect Biol 7:a016659. https:// doi.org/10.1101/cshperspect.a016659

Brown SD, Lorenz A (2016) Single-step marker switching in Schizosaccharomyces pombe using a lithium acetate transformation protocol. Bio-protocol 6:e2075. https://doi.org/10.21769/BioPr otoc. 2075

Cahoon CK, Hawley RS (2016) Regulating the construction and demolition of the synaptonemal complex. Nat Struct Mol Biol 23:369-377. https://doi.org/10.1038/nsmb.3208

Callender TL, Laureau R, Wan L et al (2016) Mek1 down regulates Rad51 activity during yeast meiosis by phosphorylation of Hed1. PLoS Genet 12:e1006226. https://doi.org/10.1371/journ al.pgen. 1006226

Carballo JA, Johnson AL, Sedgwick SG, Cha RS (2008) Phosphorylation of the axial element protein Hop1 by Mec1/Tel1 ensures 
meiotic interhomolog recombination. Cell 132:758-770. https ://doi.org/10.1016/j.cell.2008.01.035

Caryl AP, Armstrong SJ, Jones GH, Franklin FC (2000) A homologue of the yeast $H O P 1$ gene is inactivated in the Arabidopsis meiotic mutant asy1. Chromosoma 109:62-71. https://doi.org/10.1007/ s004120050413

Couteau F, Zetka M (2005) HTP-1 coordinates synaptonemal complex assembly with homolog alignment during meiosis in C. elegans. Genes Dev 19:2744-2756. https://doi.org/10.1101/gad.1348205

Craven RA, Griffiths DJ, Sheldrick KS et al (1998) Vectors for the expression of tagged proteins in Schizosaccharomyces pombe. Gene 221:59-68. https://doi.org/10.1016/S0378 -1119(98)00434-X

Daniel K, Lange J, Hached K et al (2011) Meiotic homologue alignment and its quality surveillance are controlled by mouse HORMAD1. Nat Cell Biol 13:599-610. https://doi. org/10.1038/ncb2213

de los Santos T, Hollingsworth NM (1999) Red1p, a MEK1-dependent phosphoprotein that physically interacts with Hop1p during meiosis in yeast. J Biol Chem 274:1783-1790. https://doi. org/10.1074/jbc.274.3.1783

Ding DQ, Haraguchi T, Hiraoka Y (2016) A cohesin-based structural platform supporting homologous chromosome pairing in meiosis. Curr Genet 62:499-502. https://doi.org/10.1007/ s00294-016-0570-x

Doe CL, Dixon J, Osman F, Whitby MC (2000) Partial suppression of the fission yeast rqhl(-) phenotype by expression of a bacterial Holliday junction resolvase. EMBO J 19:2751-2762. https ://doi.org/10.1093/emboj/19.11.2751

Ellermeier C, Schmidt H, Smith GR (2004) Swi5 acts in meiotic DNA joint molecule formation in Schizosaccharomyces pombe. Genetics 168:1891-1898. https://doi.org/10.1534/ genetics.104.034280

Estreicher A, Lorenz A, Loidl J (2012) Mug20, a novel protein associated with linear elements in fission yeast meiosis. Curr Genet 58:119-127. https://doi.org/10.1007/s00294-012-0369-3

Faul F, Erdfelder E, Lang A-G, Buchner A (2007) G*Power: a flexible statistical power analysis program for the social, behavioral, and biomedical sciences. Behav Res Methods 39:175-191. https:// doi.org/10.3758/BF03193146

Faul F, Erdfelder E, Buchner A, Lang A-G (2009) Statistical power analyses using $\mathrm{G}^{*}$ Power 3.1: tests for correlation and regression analyses. Behav Res Methods 41:1149-1160. https://doi. org/10.3758/BRM.41.4.1149

Flor-Parra I, Zhurinsky J, Bernal M et al (2014) A Lallzyme MMXbased rapid method for fission yeast protoplast preparation. Yeast 31:61-66. https://doi.org/10.1002/yea.2994

Fukuda T, Daniel K, Wojtasz L et al (2010) A novel mammalian HORMA domain-containing protein, HORMAD1, preferentially associates with unsynapsed meiotic chromosomes. Exp Cell Res 316:158-171. https://doi.org/10.1016/j.yexcr.2009.08.007

Goldstein AL, McCusker JH (1999) Three new dominant drug resistance cassettes for gene disruption in Saccharomyces cerevisiae. Yeast 15:1541-1553. https://doi.org/10.1002/(SICI)10970061(199910)15:14<1541::AID-YEA476>3.0.CO;2-K

Goodyer W, Kaitna S, Couteau F et al (2008) HTP-3 links DSB formation with homolog pairing and crossing over during C. elegans meiosis. Dev Cell 14:263-274. https://doi.org/10.1016/j.devce 1.2007.11.016

Gray S, Cohen PE (2016) Control of meiotic crossovers: from doublestrand break formation to designation. Annu Rev Genet 50:175210. https://doi.org/10.1146/annurev-genet-120215-035111

Grimm C, Kohli J, Murray J, Maundrell K (1988) Genetic engineering of Schizosaccharomyces pombe: a system for gene disruption and replacement using the ura 4 gene as a selectable marker. Mol Gen Genet 215:81-86. https://doi.org/10.1007/BF00331307
Grishchuk AL, Kohli J (2003) Five RecA-like proteins of Schizosaccharomyces pombe are involved in meiotic recombination. Genetics 165:1031-1043

Hartsuiker E, Neale MJ, Carr AM (2009) Distinct requirements for the Rad32(Mre11) nuclease and Ctp1(CtIP) in the removal of covalently bound topoisomerase I and II from DNA. Mol Cell 33:117-123. https://doi.org/10.1016/j.molcel.2008.11.021

Haruta N, Kurokawa Y, Murayama Y et al (2006) The Swi5-Sfr1 complex stimulates Rhp51/Rad51- and Dmc1-mediated DNA strand exchange in vitro. Nat Struct Mol Biol 13:823-830. https://doi. org/10.1038/nsmb1136

Hayase A, Takagi M, Miyazaki T et al (2004) A protein complex containing Mei5 and Sae3 promotes the assembly of the meiosisspecific RecA homolog Dmc1. Cell 119:927-940. https://doi. org/10.1016/j.cell.2004.10.031

Hollingsworth NM, Byers B (1989) HOP1: a yeast meiotic pairing gene. Genetics 121:445-462

Hollingsworth NM, Goetsch L, Byers B (1990) The HOP1 gene encodes a meiosis-specific component of yeast chromosomes. Cell 61:73-84. https://doi.org/10.1016/0092-8674(90)90216-2

Hong S, Sung Y, Yu M et al (2013) The logic and mechanism of homologous recombination partner choice. Mol Cell 51:440 453. https://doi.org/10.1016/j.molcel.2013.08.008

Hunter N (2015) Meiotic recombination: the essence of heredity. Cold Spring Harb Perspect Biol 7:a016618. https://doi.org/10.1101/ cshperspect.a016618

Hyppa RW, Smith GR (2010) Crossover invariance determined by partner choice for meiotic DNA break repair. Cell 142:243-255. https ://doi.org/10.1016/j.cell.2010.05.041

Iwasaki O, Noma K (2016) Condensin-mediated chromosome organization in fission yeast. Curr Genet 62:739-743. https://doi. org/10.1007/s00294-016-0601-7

Keeney JB, Boeke JD (1994) Efficient targeted integration at leul32 and ura4-294. in Schizosaccharomyces pombe. Genetics 136:849-856

Khan K, Madhavan TPV, Kshirsagar R et al (2013) N-terminal disordered domain of Saccharomyces cerevisiae Hop1 protein is dispensable for DNA binding, bridging, and synapsis of doublestranded DNA molecules but is necessary for spore formation. Biochemistry 52:5265-5279. https://doi.org/10.1021/bi4005528

Kim Y, Rosenberg SC, Kugel CL et al (2014) The chromosome axis controls meiotic events through a hierarchical assembly of HORMA domain proteins. Dev Cell 31:487-502. https://doi. org/10.1016/j.devcel.2014.09.013

Kogo H, Tsutsumi M, Ohye T et al (2012) HORMAD1-dependent checkpoint/surveillance mechanism eliminates asynaptic oocytes. Genes Cells 17:439-454. https://doi.org/10.111 1/j.1365-2443.2012.01600.x

Lam I, Keeney S (2015) Mechanism and regulation of meiotic recombination initiation. Cold Spring Harb Perspect Biol 7:a016634. https://doi.org/10.1101/cshperspect.a016634

Latypov V, Rothenberg M, Lorenz A et al (2010) Roles of Hop1 and Mek1 in meiotic chromosome pairing and recombination partner choice in Schizosaccharomyces pombe. Mol Cell Biol 30:15701581. https://doi.org/10.1128/MCB.00919-09

Loidl J (2006) S. pombe linear elements: the modest cousins of synaptonemal complexes. Chromosoma 115:260-271. https://doi. org/10.1007/s00412-006-0047-7

Loidl J (2016) Conservation and variability of meiosis across the eukaryotes. Annu Rev Genet 50:293-316. https://doi.org/10.1146/ annurev-genet-120215-035100

Loidl J, Lorenz A (2009) Analysis of Schizosaccharomyces pombe meiosis by nuclear spreading. Methods Mol Biol 558:15-36. https://doi.org/10.1007/978-1-60761-103-5_2 
Loidl J, Klein F, Scherthan H (1994) Homologous pairing is reduced but not abolished in asynaptic mutants of yeast. J Cell Biol 125:1191-1200. https://doi.org/10.1083/jcb.125.6.1191

Lorenz A (2015) New cassettes for single-step drug resistance and prototrophic marker switching in fission yeast. Yeast 32:703-710. https://doi.org/10.1002/yea.3097

Lorenz A (2017) Modulation of meiotic homologous recombination by DNA helicases. Yeast 34:195-203. https://doi.org/10.1002/ yea.3227

Lorenz A (2018) Supporting Online Material-Genetic interactions between the chromosome axis-associated protein Hop1 and homologous recombination determinants in Schizosaccharomyces pombe. https://doi.org/10.6084/m9.figshare.5373163.v2

Lorenz A, Wells JL, Pryce DW et al (2004) S. pombe meiotic linear elements contain proteins related to synaptonemal complex components. J Cell Sci 117:3343-3351. https://doi. org $/ 10.1242 /$ jcs.01203

Lorenz A, Estreicher A, Kohli J, Loidl J (2006) Meiotic recombination proteins localize to linear elements in Schizosaccharomyces pombe. Chromosoma 115:330-340. https://doi. org/10.1007/s00412-006-0053-9

Lorenz A, Osman F, Folkyte V et al (2009) Fbh1 limits Rad51dependent recombination at blocked replication forks. Mol Cell Biol 29:4742-4756. https://doi.org/10.1128/MCB.00471-09

Lorenz A, West SC, Whitby MC (2010) The human Holliday junction resolvase GEN1 rescues the meiotic phenotype of a Schizosaccharomyces pombe mus 81 mutant. Nucleic Acids Res 38:1866-1873. https://doi.org/10.1093/nar/gkp1179

Lorenz A, Osman F, Sun W et al (2012) The fission yeast FANCM ortholog directs non-crossover recombination during meiosis. Science 336:1585-1588. https://doi.org/10.1126/science.12201 11

Lorenz A, Mehats A, Osman F, Whitby MC (2014) Rad51/Dmc1 paralogs and mediators oppose DNA helicases to limit hybrid DNA formation and promote crossovers during meiotic recombination. Nucleic Acids Res 42:13723-13735. https://doi. org/10.1093/nar/gku1219

Martín V, Chahwan C, Gao H et al (2006) Sws1 is a conserved regulator of homologous recombination in eukaryotic cells. EMBO J 25:2564-2574. https://doi.org/10.1038/sj.emboj.7601141

Martinez-Perez E, Villeneuve AM (2005) HTP-1-dependent constraints coordinate homolog pairing and synapsis and promote chiasma formation during $C$. elegans meiosis. Genes Dev 19:2727-2743. https://doi.org/10.1101/gad.1338505

Mata J, Lyne R, Burns G, Bähler J (2002) The transcriptional program of meiosis and sporulation in fission yeast. Nat Genet 32:143-147. https://doi.org/10.1038/ng951

Matsuyama A, Arai R, Yashiroda Y et al (2006) ORFeome cloning and global analysis of protein localization in the fission yeast Schizosaccharomyces pombe. Nat Biotechnol 24:841-847. https://doi.org/10.1038/nbt1222

Mimitou EP, Symington LS (2011) DNA end resection-unraveling the tail. DNA Repair 10:344-348. https://doi.org/10.1016/j. dnarep.2010.12.004

Molnar M (2003) Linear element formation and their role in meiotic sister chromatid cohesion and chromosome pairing. J Cell Sci 116:1719-1731. https://doi.org/10.1242/jcs.00387

Moreno MB, Durán A, Ribas JC (2000) A family of multifunctional thiamine-repressible expression vectors for fission yeast. Yeast 16:861-872. https://doi.org/10.1002/10970061(20000630)16:9<861::AID-YEA577>3.0.CO;2-9

Neale MJ, Pan J, Keeney S (2005) Endonucleolytic processing of covalent protein-linked DNA double-strand breaks. Nature 436:1053-1057. https://doi.org/10.1038/nature03872

Niu H, Wan L, Baumgartner B et al (2005) Partner choice during meiosis is regulated by Hop1-promoted dimerization of Mek1.
Mol Biol Cell 16:5804-5818. https://doi.org/10.1091/mbc. E05-05-0465

Niu H, Wan L, Busygina V et al (2009) Regulation of meiotic recombination via Mek1-mediated Rad54 phosphorylation. Mol Cell 36:393-404. https://doi.org/10.1016/j.molcel.2009.09.029

Olson LW, Eden U, Egel-Mitani M, Egel R (1978) Asynaptic meiosis in fission yeast? Hereditas 89:189-199. https://doi. org/10.1111/j.1601-5223.1978.tb01275.x

Osman F, Dixon J, Doe CL, Whitby MC (2003) Generating crossovers by resolution of nicked Holliday junctions: a role for Mus81-Eme1 in meiosis. Mol Cell 12:761-774. https://doi. org/10.1016/S1097-2765(03)00343-5

Osman K, Yang J, Roitinger E et al (2018) Affinity proteomics reveals extensive phosphorylation of the Brassica chromosome axis protein ASY1 and a network of associated proteins at prophase I of meiosis. Plant J 93:17-33. https://doi.org/10.1111/tpj.13752

Pancaldi V, Saraç OS, Rallis C et al (2012) Predicting the fission yeast protein interaction network. G3 (Bethesda) 2:453-467. https:// doi.org/10.1534/g3.111.001560

Phadnis N, Cipak L, Polakova S et al (2015) Casein kinase 1 and phosphorylation of cohesin subunit Rec11 (SA3) promote meiotic recombination through linear element formation. PLoS Genet 11:e1005225. https://doi.org/10.1371/journal.pgen.1005225

Rinaldi VD, Bolcun-Filas E, Kogo $\mathrm{H}$ et al (2017) The DNA damage checkpoint eliminates mouse oocytes with chromosome synapsis failure. Mol Cell 67:1026-1036. https://doi.org/10.1016/j.molce 1.2017.07.027

Rosenberg SC, Corbett KD (2015) The multifaceted roles of the HORMA domain in cellular signaling. J Cell Biol 211:745-755. https://doi.org/10.1083/jcb.201509076

Rothenberg M, Kohli J, Ludin K (2009) Ctp1 and the MRN-complex are required for endonucleolytic Rec12 removal with release of a single class of oligonucleotides in fission yeast. PLoS Genet 5:e1000722. https://doi.org/10.1371/journal.pgen.1000722

Sabatinos SA, Forsburg SL (2010) Molecular genetics of Schizosaccharomyces pombe. Methods Enzymol 470:759-795. https://doi. org/10.1016/S0076-6879(10)70032-X

Sakuno T, Watanabe Y (2015) Phosphorylation of cohesin Rec11/SA3 by Casein Kinase 1 promotes homologous recombination by assembling the meiotic chromosome axis. Dev Cell 32:220-230. https://doi.org/10.1016/j.devcel.2014.11.033

Sambrook JF, Russell DW (2000) Molecular cloning: a laboratory manual, 3rd edn. Cold Spring Harbor Laboratory Press, Cold Spring Harbor

Sanchez-Moran E, Santos J-L, Jones GH, Franklin FCH (2007) ASY1 mediates AtDMC1-dependent interhomolog recombination during meiosis in Arabidopsis. Genes Dev 21:2220-2233. https:// doi.org/10.1101/gad.439007

Sasanuma H, Tawaramoto MS, Lao JP et al (2013) A new protein complex promoting the assembly of Rad51 filaments. Nat Commun 4:1676. https://doi.org/10.1038/ncomms 2678

Sato M, Dhut S, Toda T (2005) New drug-resistant cassettes for gene disruption and epitope tagging in Schizosaccharomyces pombe. Yeast 22:583-591. https://doi.org/10.1002/yea.1233

Schwacha A, Kleckner N (1997) Interhomolog bias during meiotic recombination: meiotic functions promote a highly differentiated interhomolog-only pathway. Cell 90:1123-1135. https://doi. org/10.1016/S0092-8674(00)80378-5

Shinohara A, Gasior S, Ogawa T et al (1997) Saccharomyces cerevisiae recA homologues $R A D 51$ and $D M C 1$ have both distinct and overlapping roles in meiotic recombination. Genes Cells 2:615-629. https://doi.org/10.1046/j.1365-2443.1997.1480347.x

Sievers F, Wilm A, Dineen D et al (2014) Fast, scalable generation of high-quality protein multiple sequence alignments using Clustal Omega. Mol Syst Biol 7:539-539. https://doi.org/10.1038/ msb.2011.75 
Silva N, Ferrandiz N, Barroso C et al (2014) The fidelity of synaptonemal complex assembly is regulated by a signaling mechanism that controls early meiotic progression. Dev Cell 31:503-511. https://doi.org/10.1016/j.devcel.2014.10.001

Smith GR (2009) Genetic analysis of meiotic recombination in Schizosaccharomyces pombe. Methods Mol Biol 557:65-76. https://doi. org/10.1007/978-1-59745-527-5_6

Smith AV, Roeder GS (1997) The yeast Red1 protein localizes to the cores of meiotic chromosomes. J Cell Biol 136:957-967. https ://doi.org/10.1083/jcb.136.5.957

Smith GR, Boddy MN, Shanahan P, Russell P (2003) Fission yeast Mus81.Eme1 Holliday junction resolvase is required for meiotic crossing over but not for gene conversion. Genetics 165:2289-2293

Spirek M, Estreicher A, Csaszar E et al (2010) SUMOylation is required for normal development of linear elements and wildtype meiotic recombination in Schizosaccharomyces pombe. Chromosoma 119:59-72. https://doi.org/10.1007/s0041 2-009-0241-5

Stanzione M, Baumann M, Papanikos F et al (2016) Meiotic DNA break formation requires the unsynapsed chromosome axis-binding protein IHO1 (CCDC36) in mice. Nat Cell Biol 18:12081220. https://doi.org/10.1038/ncb3417

Sung P (1997) Yeast Rad55 and Rad57 proteins form a heterodimer that functions with replication protein A to promote DNA strand exchange by Rad51 recombinase. Genes Dev 11:1111-1121. https://doi.org/10.1101/gad.11.9.1111

Tripathi P, Pal D, Muniyappa K (2007) Saccharomyces cerevisiae Hop1 protein zinc finger motif binds to the Holliday junction and distorts the DNA structure: implications for Holliday junction migration. Biochemistry 46:12530-12542. https://doi. org/10.1021/bi701078v
Tsubouchi H, Roeder GS (2006) Budding yeast Hed1 down-regulates the mitotic recombination machinery when meiotic recombination is impaired. Genes Dev 20:1766-1775. https://doi. org/10.1101/gad.1422506

Tsutsui Y, Khasanov FK, Shinagawa H et al (2001) Multiple interactions among the components of the recombinational DNA repair system in Schizosaccharomyces pombe. Genetics 159:91-105

Vitelli F, Piccini M, Caroli F et al (1999) Identification and characterization of a highly conserved protein absent in the Alport syndrome (A), mental retardation (M), midface hypoplasia (M), and elliptocytosis (E) contiguous gene deletion syndrome (AMME). Genomics 55:335-340. https://doi.org/10.1006/geno.1998.5666

West AMV, Komives EA, Corbett KD (2017) Conformational dynamics of the Hop1 HORMA domain reveal a common mechanism with the spindle checkpoint protein Mad2. Nucleic Acids Res. https://doi.org/10.1093/nar/gkx1196

Woltering D, Baumgartner B, Bagchi S et al (2000) Meiotic segregation, synapsis, and recombination checkpoint functions require physical interaction between the chromosomal proteins Red1p and Hop1p. Mol Cell Biol 20:6646-6658. https://doi. org/10.1128/MCB.20.18.6646-6658.2000

Young JA, Hyppa RW, Smith GR (2004) Conserved and nonconserved proteins for meiotic DNA breakage and repair in yeasts. Genetics 167:593-605. https://doi.org/10.1534/genetics.103.023762

Zetka MC, Kawasaki I, Strome S, Müller F (1999) Synapsis and chiasma formation in Caenorhabditis elegans require HIM-3, a meiotic chromosome core component that functions in chromosome segregation. Genes Dev 13:2258-2270. https://doi.org/10.1101/ gad.13.17.2258 